\title{
Renormalized Energy and Forces on Dislocations
}

\author{
Paolo Cermelli Giovanni Leoni
}

January 10, 2005

\begin{abstract}
In this work we discuss, from a variational viewpoint, the equilibrium problem for a finite number of Volterra dislocations in a plane domain. For a given set of singularities at fixed locations, we characterize elastic equilibrium as the limit of the minimizers of a family of energy functionals, obtained by a finite-core regularization of the elastic-energy functional. We give a sharp asymptotic estimate of the minimum energy as the core radius tends to zero, which allows to eliminate this internal length scale from the problem. The energy content of a set of dislocations is fully characterized by the regular part of the asymptotic expansion, the so-called renormalized energy, which contains all information regarding self- and mutual interactions between the defects. Thus our result may be considered as the analogue for dislocations of the classical result of Bethuel, Brezis and Hélein for Ginzburg-Landau vortices. We view the renormalized energy as the basic tool for the study of the discrete-to-continuum limit in plasticity of crystals, i.e., the passage from models of isolated defects to theories of continuous distributions of dislocations. The renormalized energy is a function of the defect positions only: we prove that its derivative with respect to the position of a given dislocation is the resultant of the Eshelby stress on that dislocation, which can be identified in turn with the classical Peach-Köhler force.
\end{abstract}

Key words. dislocations, variational methods, variational techniques for singularities, forces on defects

AMS subject classifications. 35J50 Variational methods for elliptic systems, 74G65 Energy minimization, 74G70 Stress concentrations, singularities,

\section{Introduction}

Dislocations are the most common and important defects in crystals, and influence their behavior in multiple ways: for instance, isolated dislocations generate concentrations of stress which affect the chemical and electronic properties of solids, while the collective glide motion of large sets of dislocations represents the basic mechanism for plastic slip in ductile solids (cf., e.g., [3], [4], [20], [28], [35], [38] and [39]).

Hence, it is of considerable interest to study the behavior both of isolated and large sets of dislocations.

However, the study of isolated dislocations and that of large clusters of defects require completely different mathematical models and techniques. Problems involving isolated defects involve scales which are typically of the order of the Burgers vector, which is in turn of the order of the interatomic distance in a crystal: for instance, the energy of a set of dislocation scales as $\ln (1 / \epsilon)$, with $\epsilon$ the radius of the dislocation core (usually chosen to be of order of the Burgers vector), while the Peierls-Nabarro force acts on distances of order of a few atomic spacings. 
On the other hand, the characteristic scales involved in the phenomena related to the collective behavior of large clusters of dislocations, typically in plasticity, are much larger: the paradigmatic example being the self-organization of stored dislocations in cell patterns, typical of hardening stage II [31]. In this case, the characteristic distance between the cell walls is macroscopic, much larger than the interatomic distances. Such problems must be studied in terms of dislocation densities, rather than in terms of isolated dislocations, and require the introduction, in the expression for the macroscopic energy of the solid, of terms which depend on the plastic strain gradients ([1], [17], [18], [19], [23], [24], [25], [30], [36]). These terms are necessarily phenomenological - for instance, energies are assumed to be quadratic in the plastic strain gradients, and so on. However, such simple choices often lead to unphysical behavior, as shown in [9] for interfacial dislocations in epitaxial films.

At a still larger scale lives classical plasticity: here plastic strain gradients are ignored, no internal length scale is introduced, and dislocations do not appear explicitly in the model: such models of course are not capable of describing self-organization of defects in regular patterns.

Hence, a major open problem in the theory of defects in solids is to correlate the microscopic (isolated defects) and the macroscopic (gradient theories) approaches. Specifically, it would be useful to develop a theoretical framework which allows to characterize the constitutive relations of the continuum models, using the information gained by "ab initio" models of finite sets of dislocations.

The goal of this paper may be viewed as the first stage of this project: we give a variational formulation of the equilibrium problem for a finite number of dislocations in a plane domain, and characterize the energy content of a body with isolated defects in terms of a regular function of the defect configuration, the so-called renormalized energy.

Precisely, consider a finite number of dislocations in an elastic solid: since the stress field induced by a dislocation is short ranged, it is reasonable to work in the approximation of linear elasticity, which may be assumed to be valid sufficiently far from the defect (this topic has been studied extensively in the literature, and explicit solutions are known in special cases ([34], [28], [35])). We restrict attention to plane isotropic elasticity ${ }^{1}$. Let $\Omega$ be a regular domain in $\mathbb{R}^{2}$ : in linear elasticity, a displacement of $\Omega$ is a regular vector field $\boldsymbol{u}$ on $\Omega$, with gradient $\nabla \boldsymbol{u}=\boldsymbol{H}$. The equilibrium equations have the form $\operatorname{Div} C[\boldsymbol{E}(\boldsymbol{u})]=0$, with $C$ a linear operator from $\mathbb{R}^{2 \times 2}$ into itself, and $\boldsymbol{E}(\boldsymbol{u})=\frac{1}{2}\left(\nabla \boldsymbol{u}+(\nabla \boldsymbol{u})^{\top}\right)$ the infinitesimal strain tensor.

In this framework, Volterra dislocations may be viewed as singularities of the field $\boldsymbol{H}$. Precisely, fix a finite set of points $\left\{\boldsymbol{x}_{1}, \ldots, \boldsymbol{x}_{N}\right\}$ in $\Omega$, and a set of vectors $\left\{\boldsymbol{b}_{1}, \ldots, \boldsymbol{b}_{N}\right\}$, with $\boldsymbol{b}_{i} \in \mathbb{R}^{2}$ : we say that a tensor field $\boldsymbol{H}$ on $\Omega \backslash\left\{\boldsymbol{x}_{1}, \ldots, \boldsymbol{x}_{N}\right\}$ corresponds to a system of dislocations located at $\left\{\boldsymbol{x}_{1}, \ldots, \boldsymbol{x}_{N}\right\}$ with Burgers vectors $\left\{\boldsymbol{b}_{1}, \ldots, \boldsymbol{b}_{N}\right\}$, if

$$
\left\{\begin{array}{l}
\operatorname{Curl} \boldsymbol{H}=\sum_{i=1}^{N} \boldsymbol{b}_{i} \delta_{\boldsymbol{x}_{i}} \\
\operatorname{Div} C[\boldsymbol{E}(\boldsymbol{H})]=0
\end{array} \quad \text { in } \Omega\right.
$$

in the sense of distributions, where $\boldsymbol{E}(\boldsymbol{H})=\frac{1}{2}\left(\boldsymbol{H}+\boldsymbol{H}^{\top}\right)$ is the strain associated to $\boldsymbol{H}$.

Solutions of (1.1) are not unique even modulo an infinitesimal rigid motion and, moreover, no variational principle may be associated to (1.1), since the elastic energy of a system of Volterra dislocations is not finite.

Hence, it is necessary to regularize the theory by removing a core $B_{\varepsilon}\left(\boldsymbol{x}_{i}\right)$ of radius $\varepsilon$ around each

\footnotetext{
${ }^{1}$ Point defects in plane elasticity may be effectively used to model straight edge dislocations orthogonal to the plane of strain.
} 
dislocation; letting $\Omega_{\varepsilon}=\Omega \backslash\left(\cup_{i=1}^{N} B_{\varepsilon}\left(\boldsymbol{x}_{i}\right)\right)$, we solve the family of minimization problems

$$
\left.\min _{\boldsymbol{H} \in H\left(\boldsymbol{b}_{1}, \ldots, \boldsymbol{b}_{N} ; \Omega_{\varepsilon}\right)} \int_{\Omega_{\varepsilon}} W(\boldsymbol{E}(\boldsymbol{H}))\right) d a,
$$

where $W(\boldsymbol{E})=\frac{1}{2} \boldsymbol{E} \cdot C[\boldsymbol{E}]$ is the elastic energy density,

$$
H\left(\boldsymbol{b}_{1}, \ldots, \boldsymbol{b}_{N} ; \Omega_{\varepsilon}\right)=\left\{\boldsymbol{H} \in H\left(\operatorname{Curl} 0 ; \Omega_{\varepsilon}\right): \int_{\partial B_{\varepsilon}\left(\boldsymbol{x}_{i}\right)} \boldsymbol{H} \boldsymbol{t} d s=\boldsymbol{b}_{i}, \quad i=1, \ldots, N\right\},
$$

and $\boldsymbol{t}$ is the unit tangent vector to $\partial B_{\varepsilon}\left(\boldsymbol{x}_{i}\right)$.

Our first result show that the solutions $\boldsymbol{H}_{\varepsilon}$ of (1.2) converge strongly in $L_{\text {loc }}^{2}\left(\Omega \backslash \cup_{i=1}^{N}\left\{\boldsymbol{x}_{i}\right\} ; \mathbb{R}^{2 \times 2}\right)$, as $\varepsilon \rightarrow 0$, to a solution $\boldsymbol{H}_{0}$ of (1.1). This solution is unique modulo a rigid motion. More precisely, we show that

$$
\boldsymbol{H}_{\varepsilon} \rightarrow \boldsymbol{H}_{0}=\sum_{i=1}^{N} \boldsymbol{K}_{i}+\nabla \boldsymbol{u}_{0}
$$

where $\boldsymbol{K}_{i}$ are distributional solutions of $\operatorname{Curl} \boldsymbol{K}_{i}=\boldsymbol{b}_{i} \delta_{\boldsymbol{x}_{i}}$ on $\mathbb{R}^{2}$, and $\boldsymbol{u}_{0} \in H^{1}\left(\Omega ; \mathbb{R}^{2}\right)$ is a regular displacement field which is a minimizer of the functional

$$
I_{0}(\boldsymbol{u}):=\int_{\Omega} W(\boldsymbol{E}(\boldsymbol{u})) d a+\sum_{i=1}^{N} \int_{\partial \Omega} \boldsymbol{u} \cdot C\left[\boldsymbol{E}\left(\boldsymbol{K}_{i}\right)\right] \boldsymbol{n} d s,
$$

on $H^{1}\left(\Omega ; \mathbb{R}^{2}\right)$.

The field $\boldsymbol{H}_{0}$ is independent of the internal length scale $\varepsilon$, but its energy is not finite: we obtain a sharp asymptotic estimate as $\varepsilon \rightarrow 0$ for the minimum energy in (1.2), of the form

$$
\left.\int_{\Omega_{\varepsilon}} W\left(\boldsymbol{E}\left(\boldsymbol{H}_{\varepsilon}\right)\right)\right) d a=\sum_{i=1}^{N} \frac{\mu(\lambda+\mu)}{4 \pi(\lambda+2 \mu)}\left|\boldsymbol{b}_{i}\right|^{2} \ln \frac{1}{\varepsilon}+F\left(\boldsymbol{x}_{1}, \ldots, \boldsymbol{x}_{N}\right)+O(\varepsilon)+\text { Const. },
$$

where $\lambda, \mu$ are the Lamé moduli, and

$$
F\left(\boldsymbol{x}_{1}, \ldots, \boldsymbol{x}_{N}\right)=F_{\text {self }}\left(\boldsymbol{x}_{1}, \ldots, \boldsymbol{x}_{N}\right)+F_{\text {int }}\left(\boldsymbol{x}_{1}, \ldots, \boldsymbol{x}_{N}\right)+F_{\text {elastic }}\left(\boldsymbol{x}_{1}, \ldots, \boldsymbol{x}_{N}\right),
$$

is the renormalized energy, with

$$
\left\{\begin{array}{l}
F_{\text {self }}\left(\boldsymbol{x}_{1}, \ldots, \boldsymbol{x}_{N}\right)=\sum_{i=1}^{N} \int_{\Omega \backslash B_{R}\left(\boldsymbol{x}_{i}\right)} W\left(\boldsymbol{E}\left(\boldsymbol{K}_{i}\right)\right) d a+\sum_{i=1}^{N} \frac{\mu(\lambda+\mu)}{4 \pi(\lambda+2 \mu)}\left|\boldsymbol{b}_{i}\right|^{2} \ln R, \\
F_{\text {int }}\left(\boldsymbol{x}_{1}, \ldots, \boldsymbol{x}_{N}\right)=\sum_{i=1}^{N-1} \sum_{j=i+1}^{N} \int_{\Omega} C\left[\boldsymbol{E}\left(\boldsymbol{K}_{i}\right)\right] \cdot \boldsymbol{E}\left(\boldsymbol{K}_{j}\right) d a, \\
F_{\text {elastic }}\left(\boldsymbol{x}_{1}, \ldots, \boldsymbol{x}_{N}\right)=\int_{\Omega} W\left(\boldsymbol{E}\left(\boldsymbol{u}_{0}\right)\right) d a+\sum_{i=1}^{N} \int_{\partial \Omega} \boldsymbol{u}_{0} \cdot C\left[\boldsymbol{E}\left(\boldsymbol{K}_{i}\right)\right] \boldsymbol{n} d s,
\end{array}\right.
$$

and

$$
R=\frac{1}{4} \min \left(\left\{\left|\boldsymbol{x}_{i}-\boldsymbol{x}_{j}\right|: i, j=1, \ldots, N, i \neq j\right\} \cup\left\{\operatorname{dist}\left(\left\{\boldsymbol{x}_{1}, \ldots, \boldsymbol{x}_{N}\right\}, \partial \Omega\right)\right\}\right) .
$$


It is important to remark that while for special domains the asymptotic formula

$$
\left.\int_{\Omega_{\varepsilon}} W\left(\boldsymbol{E}\left(\boldsymbol{H}_{\varepsilon}\right)\right)\right) d a \sim \sum_{i=1}^{N} \frac{\mu(\lambda+\mu)}{4 \pi(\lambda+2 \mu)}\left|\boldsymbol{b}_{i}\right|^{2} \ln \frac{1}{\varepsilon}
$$

is classical (see e.g. [28, 35]) and can be obtained by solving explicitly the Euler equation (1.1) (see [34]), for general domains there are various formal arguments in support of (1.8) but we are not aware of any rigourous derivation prior to ours.

More importantly, the introduction of the renormalized energy in this context appears to be new, and thus our result may be considered as the analogue for dislocations of the classical result of Bethuel, Brezis and Hélein (see Chapter 2 in [6], see also ) for Ginzburg-Landau vortices. We refer to the monograph [6] for more details about the Ginzburg-Landau functional (see also [2, 7, 27] and the references contained therein for more recent results).

Note that the renormalized energy is independent of the core radius, and is a function of the defect position which fully characterizes the energy content of a dislocated body. Hence, it provides a basis for the study of the behavior of finite sets of dislocations.

As an example application of these ideas, we prove that the interaction energy $F_{\text {int }}$ in $(1.7)_{2}$ diverges logarithmically with the relative distance between the defects:

$$
F_{\mathrm{int}}\left(\boldsymbol{x}_{1}, \ldots, \boldsymbol{x}_{N}\right)=\sum_{i=1}^{N-1} \sum_{j=i+1}^{N} \frac{\mu(\lambda+\mu)}{\pi(\lambda+2 \mu)} \boldsymbol{b}_{i} \cdot \boldsymbol{b}_{j} \ln \frac{1}{\left|\boldsymbol{x}_{i}-\boldsymbol{x}_{j}\right|}+O(1)
$$

as $\left|\boldsymbol{x}_{i}-\boldsymbol{x}_{j}\right| \rightarrow 0$.

When more than one dislocation is present, or an external stress is applied to the dislocated body, defects interact between themselves and with the applied field, by means of the so-called Peach-Köhler force [37]. Since the renormalized energy contains all the information about defect interactions, a natural question is whether it is somehow related to the Peach-Köhler force on dislocations. Indeed, the asymptotic analysis of a regularized Ginzburg-Landau equation, intended to model disclinations in liquid crystals, shows that on a long time scale defects move according to a simple evolution equation, which has the form velocity $=$ force on the defect $([26],[33],[32])$, where the force on the defect is defined as the derivative of the renormalized energy with respect to the defect position.

In this line of thought, we prove the fundamental relation

$$
\nabla_{\boldsymbol{x}_{k}} F=-\int_{\partial B_{R}\left(\boldsymbol{x}_{k}\right)}\left\{W\left(\boldsymbol{E}\left(\boldsymbol{H}_{0}\right)\right) \mathbf{1}-\boldsymbol{H}_{0}^{\top} C\left[\boldsymbol{E}\left(\boldsymbol{H}_{0}\right)\right]\right\} \boldsymbol{n} d s
$$

for $R<\min _{i} \frac{1}{2} \mathrm{~d}\left(\boldsymbol{x}_{i}, \partial \Omega\right)$, where the integrand $\boldsymbol{C}=W\left(\boldsymbol{E}\left(\boldsymbol{H}_{0}\right)\right) \mathbf{1}-\boldsymbol{H}_{0}^{\top} C\left[\boldsymbol{E}\left(\boldsymbol{H}_{0}\right)\right]$ is called the Eshelby stress. This object, also known as configurational stress, is usually introduced in continuum mechanics in conjunction with an additional balance law, the configurational balance, when defective structures such as interfaces, cracks or inclusions, are present [23]. The configurational balance governs the evolution of the defect, and the resultant of the Eshelby stress in (1.9) may be identified with the force acting on a defect. In the theory of elastic dislocations, the force on a defect is defined by means of the so-called Peach-Köhler force, and indeed it can be shown that the resultant of the configurational stress coincides with the Peach-Köhler force on a dislocation [8].

Hence, (1.9) shows that the derivative of the renormalized energy coincides with the force on a dislocation. 
The idea that the force on a defect is the derivative of the minimum energy with respect to changes of the defect position is the basis of Eshelby's treatment of defects ([14], [15], [13]). However, when dislocations are present the energy is not finite, so that Eshelby's approach fails without modifications: our result may be viewed as the generalization of Eshelby's notion of force on a defect, when bad singularities are associated to the defect itself.

\section{The variational problem}

Let $\Omega \subset \mathbb{R}^{2}$ be a bounded open domain with smooth boundary $\partial \Omega$, with outward unit normal $\boldsymbol{n}$. In the absence of defect, we denote by $\boldsymbol{u}: \Omega \rightarrow \mathbb{R}^{2}$ the displacement field, with displacement gradient $\nabla \boldsymbol{u}$ and strain tensor $\boldsymbol{E}(\boldsymbol{u})=\frac{1}{2}\left(\nabla \boldsymbol{u}+(\nabla \boldsymbol{u})^{\top}\right)$. We write

$$
\boldsymbol{T}=C[\boldsymbol{E}]
$$

for the (symmetric) Cauchy stress, with $C:$ Sym $\rightarrow$ Sym the elasticity tensor, a symmetric ${ }^{2}$ linear map on the space Sym of symmetric $2 \times 2$ tensors. For isotropic materials, the stress has the form

$$
C[\boldsymbol{E}]=\lambda(\operatorname{tr} \boldsymbol{E}) \mathbf{1}+2 \mu \boldsymbol{E},
$$

with $\lambda, \mu$ the Lamé moduli. The associated energy functional is

$$
J(\boldsymbol{u})=\int_{\Omega} W(\boldsymbol{E}(\boldsymbol{u})) d a
$$

which is defined on $H^{1}\left(\Omega ; \mathbb{R}^{2}\right)$. Here $W(\boldsymbol{E})=\frac{1}{2} \boldsymbol{E} \cdot C[\boldsymbol{E}]$ is the strain energy density, and we assume that the elasticity tensor $C$ is positive definite. ${ }^{3}$ In plane elasticity and for isotropic materials this is equivalent to requiring

$$
\mu>0 \text { and } \lambda+\mu>0
$$

In this paper we shall only deal with isotropic materials.

When defects such as dislocations are present, the displacement field is not single-valued, and the equilibrium problem must be formulated in terms of a $2 \times 2$ tensor field $\boldsymbol{H}$, defined away from the defects, and such that Curl $\boldsymbol{H}=0$. The field $\boldsymbol{H}$ plays the role of displacement gradient but is not necessarily the gradient of a displacement field globally defined on $\Omega$ : we will continue to use the denomination strain tensor associated to $\boldsymbol{H}$ for the symmetric part of $\boldsymbol{H}$, writing

$$
\boldsymbol{E}(\boldsymbol{H})=\frac{1}{2}\left(\boldsymbol{H}+\boldsymbol{H}^{\top}\right)
$$

More precisely, we are interested in situations in which the field $\boldsymbol{H}$ has a finite number of singularities in $\Omega$ : to this purpose, let $\left\{\boldsymbol{x}_{i}\right\}_{i=1, \ldots, N}$ be a finite sequence of points in $\Omega$, and for $\varepsilon>0$ let

$$
\Omega_{\varepsilon}=\Omega \backslash\left(\bigcup_{i=1}^{N} B_{\varepsilon}\left(\boldsymbol{x}_{i}\right)\right),
$$

\footnotetext{
${ }^{2}$ I.e., $\boldsymbol{E} \cdot C[\boldsymbol{F}]=\boldsymbol{F} \cdot C[\boldsymbol{E}]$ for any $\boldsymbol{E}, \boldsymbol{F} \in \mathrm{Sym}$, where $\cdot$ is the inner product of $2 \times 2$ tensors.

${ }^{3}$ Which implies that there exist constants $C_{1}, C_{2}>0$ such that $C_{1}|\boldsymbol{E}|^{2} \leq W(\boldsymbol{E}) \leq C_{2}|\boldsymbol{E}|^{2}$ for any $\boldsymbol{E} \in$ Sym.
} 
and consider the space ${ }^{4}$

$$
H\left(\mathrm{Curl} ; \Omega_{\varepsilon}\right):=\left\{\boldsymbol{H} \in L^{2}\left(\Omega_{\varepsilon} ; \mathbb{R}^{2 \times 2}\right): \operatorname{Curl} \boldsymbol{H} \in L^{2}\left(\Omega_{\varepsilon} ; \mathbb{R}^{2 \times 2}\right)\right\} .
$$

Following [12] we set

$$
H\left(\operatorname{Curl} 0 ; \Omega_{\varepsilon}\right):=\left\{\boldsymbol{H} \in H\left(\operatorname{Curl} ; \Omega_{\varepsilon}\right): \operatorname{Curl} \boldsymbol{H}=\mathbf{0}\right\} .
$$

We say that $\boldsymbol{H} \in H\left(\operatorname{Curl} 0 ; \Omega_{\varepsilon}\right)$ corresponds to a system of dislocations located at $\boldsymbol{x}_{i}$, with Burgers vectors $\boldsymbol{b}_{i}$ and cores $B_{\varepsilon}\left(\boldsymbol{x}_{i}\right)$, if

$$
\int_{\partial B_{\varepsilon}\left(\boldsymbol{x}_{i}\right)} \boldsymbol{H} \boldsymbol{t} d s=\boldsymbol{b}_{i}, \quad i=1, \ldots, N,
$$

with $\boldsymbol{t}$ the unit tangent to $\partial B_{\varepsilon}\left(\boldsymbol{x}_{i}\right)$. Here we have used the fact that the trace map

$$
H \mapsto H t
$$

defined on $C^{\infty}\left(\overline{\Omega_{\varepsilon}} ; \mathbb{R}^{2 \times 2}\right)$ extends by continuity to a continuous linear mapping, still denoted $\boldsymbol{H} \boldsymbol{t}$, from $H\left(\mathrm{Curl} ; \Omega_{\varepsilon}\right)$ to $H^{-\frac{1}{2}}\left(\Omega_{\varepsilon} ; \mathbb{R}^{2 \times 2}\right)$, (see e.g. Thm. 2 page 204 in [12]).

We shall denote by $H\left(\boldsymbol{b}_{1}, \ldots, \boldsymbol{b}_{N} ; \Omega_{\varepsilon}\right)$ the closed subspace of $H\left(\operatorname{Curl} 0 ; \Omega_{\varepsilon}\right)$ of tensor fields corresponding to systems of dislocations with Burgers vectors $\boldsymbol{b}_{i}$, i.e.,

$$
H\left(\boldsymbol{b}_{1}, \ldots, \boldsymbol{b}_{N} ; \Omega_{\varepsilon}\right):=\left\{\boldsymbol{H} \in H\left(\operatorname{Curl} 0 ; \Omega_{\varepsilon}\right): \int_{\partial B_{\varepsilon}\left(\boldsymbol{x}_{i}\right)} \boldsymbol{H} \boldsymbol{t} d s=\boldsymbol{b}_{i}, \quad i=1, \ldots, N\right\} .
$$

The strain energy functional is defined as in the absence of defects (cf. (2.2)),

$$
J_{\varepsilon}(\boldsymbol{H})=\int_{\Omega_{\varepsilon}} W(\boldsymbol{E}(\boldsymbol{H})) d a,
$$

and the associated minimization problem is

$\left(M_{\boldsymbol{H}, \varepsilon}\right)$ : Minimize the strain energy functional over all systems of dislocations located at given points $\left(\boldsymbol{x}_{1}, \ldots, \boldsymbol{x}_{N}\right)$, and with given Burgers vectors $\left(\boldsymbol{b}_{1}, \ldots, \boldsymbol{b}_{N}\right)$, i.e., find the solutions of

$$
\min _{\boldsymbol{H} \in H\left(\boldsymbol{b}_{1}, \ldots, \boldsymbol{b}_{N} ; \Omega_{\varepsilon}\right)} J_{\varepsilon}(\boldsymbol{H}) .
$$

Proposition 2.1. Assume that the elasticity tensor C satisfies condition (2.3). Then

$$
\boldsymbol{H}_{\varepsilon} \in H\left(\boldsymbol{b}_{1}, \ldots, \boldsymbol{b}_{N} ; \Omega_{\varepsilon}\right)
$$

is a minimizer of (2.9) if and only if $\boldsymbol{H}_{\varepsilon}$ is a weak solution of the Neumann boundary problem

$$
\begin{cases}\operatorname{Div} C\left[\boldsymbol{E}\left(\boldsymbol{H}_{\varepsilon}\right)\right]=\mathbf{0}, & \text { in } \Omega_{\varepsilon}, \\ C\left[\boldsymbol{E}\left(\boldsymbol{H}_{\varepsilon}\right)\right] \boldsymbol{n}=0, & \text { on } \partial \Omega_{\varepsilon}=\partial \Omega \cup\left(\cup_{i=1}^{N} \partial B_{\varepsilon}\left(\boldsymbol{x}_{i}\right)\right) .\end{cases}
$$

Moreover $\boldsymbol{H}_{\varepsilon}$ is unique modulo an infinitesimal rigid-body motion.

\footnotetext{
${ }^{4}$ The Curl of a two dimensional tensor field $\boldsymbol{H}$ is the vector field whose cartesian components are $(\mathrm{Curl} \boldsymbol{H})_{i}=\left(\partial_{1} H_{i 2}-\right.$ $\left.\partial_{2} H_{i 1}\right)$.
} 
Proof. Since $J_{\varepsilon}$ is quadratic it follows from standard arguments in the Calculus of Variations that $\boldsymbol{H}_{\varepsilon}$ is a minimizer if and only if satisfies the weak Euler equation

$$
\int_{\Omega_{\varepsilon}} C\left[\boldsymbol{H}_{\varepsilon}\right] \cdot \nabla \boldsymbol{w} d a=0, \quad \text { for all } \boldsymbol{w} \in H^{1}\left(\Omega_{\varepsilon} ; \mathbb{R}^{2}\right) .
$$

Indeed, note that, for every $\boldsymbol{H}, \tilde{\boldsymbol{H}} \in H\left(\boldsymbol{b}_{1}, \ldots, \boldsymbol{b}_{N} ; \Omega_{\varepsilon}\right)$, there exists $\boldsymbol{w} \in H^{1}\left(\Omega_{\varepsilon} ; \mathbb{R}^{2}\right)$ such that $\tilde{\boldsymbol{H}}=$ $\boldsymbol{H}+\nabla \boldsymbol{w}$ : moreover, for $t$ a real parameter,

$$
J_{\varepsilon}(\boldsymbol{H}+t \nabla \boldsymbol{w})-J_{\varepsilon}(\boldsymbol{H})=t \int_{\Omega_{\varepsilon}} C[\boldsymbol{H}] \cdot \nabla \boldsymbol{w} d a+t^{2} J_{\varepsilon}(\nabla \boldsymbol{w}),
$$

and this proves the assertion.

To prove uniqueness let $\boldsymbol{H}_{\varepsilon}$ and $\boldsymbol{H}_{\varepsilon}^{\prime}$ be two solutions of $(2.11)$, then $\boldsymbol{H}_{\varepsilon}^{\prime}=\boldsymbol{H}_{\varepsilon}+\boldsymbol{W}$, with $\boldsymbol{W}$ a constant skew-symmetric tensor: indeed, since $\boldsymbol{H}_{\varepsilon}$ and $\boldsymbol{H}_{\varepsilon}^{\prime}$ both belong to $H\left(\boldsymbol{b}_{1}, \ldots, \boldsymbol{b}_{N} ; \Omega_{\varepsilon}\right)$, then $\boldsymbol{H}_{\varepsilon}^{\prime}=\boldsymbol{H}_{\varepsilon}+\nabla \boldsymbol{u}$ for some $\boldsymbol{u} \in H^{1}\left(\Omega_{\varepsilon} ; \mathbb{R}^{2}\right)$, which satisfies the equation

$$
\int_{\Omega_{\varepsilon}} C[\boldsymbol{E}(\boldsymbol{u})] \cdot \nabla \boldsymbol{w} d a=0, \quad \text { for all } \boldsymbol{w} \in H^{1}\left(\Omega_{\varepsilon} ; \mathbb{R}^{2}\right) ;
$$

choosing $\boldsymbol{w}=\boldsymbol{u}$ and using the strong ellipticity of $C$, this implies that $\boldsymbol{E}(\boldsymbol{u})=0$, and, in turn, that $\boldsymbol{u}(\boldsymbol{x})=\boldsymbol{a}+\boldsymbol{W} \boldsymbol{x}$, with $\boldsymbol{a}$ and $\boldsymbol{W}$ a constant vector and a constant skew-symmetric tensor respectively, which proves the assertion.

Remark 2.2. Uniqueness of the solution of (2.9) is guaranteed for instance by assuming that the total infinitesimal rotation of the body vanishes, i.e.,

$$
\int_{\Omega_{\varepsilon}}\left(\boldsymbol{H}_{\varepsilon}-\boldsymbol{H}_{\varepsilon}^{\top}\right) d a=0
$$

\section{Existence for a single dislocation in a ball}

In this section we consider the special case where

$$
\Omega=B_{R}\left(\boldsymbol{x}_{0}\right)
$$

and we have a single dislocation located at $\boldsymbol{x}_{0}$ and with Burger vector $\boldsymbol{b}$. We are interested in the asymptotic behavior as $\varepsilon \rightarrow 0^{+}$and $R \rightarrow \infty$ of the solutions of the minimization problem

$$
\left.\min _{\boldsymbol{H} \in H\left(\boldsymbol{b} ; B_{R}\left(\boldsymbol{x}_{0}\right) \backslash B_{\varepsilon}\left(\boldsymbol{x}_{0}\right)\right)} \int_{B_{R}\left(\boldsymbol{x}_{0}\right) \backslash B_{\varepsilon}\left(\boldsymbol{x}_{0}\right)} W(\boldsymbol{E}(\boldsymbol{H}))\right) d a .
$$

Proposition 3.1. Assume that the elasticity tensor $C$ satisfies condition (2.3) and let $\boldsymbol{K}_{\boldsymbol{b}, \varepsilon, R}$ be the unique solution of (3.1) such that

$$
\int_{B_{R}\left(\boldsymbol{x}_{0}\right) \backslash B_{\varepsilon}\left(\boldsymbol{x}_{0}\right)}\left(\boldsymbol{K}_{\boldsymbol{b}, \varepsilon, R}-\boldsymbol{K}_{\boldsymbol{b}, \varepsilon, R}^{\top}\right) d a=0 .
$$


Then $\left\{\boldsymbol{K}_{\boldsymbol{b}, \varepsilon, R}\right\}$ converges uniformly on compact subsets of $\mathbb{R}^{2} \backslash\left\{\boldsymbol{x}_{0}\right\}$ as $\varepsilon \rightarrow 0$ and $R \rightarrow \infty$ to the function

$$
\boldsymbol{K}_{\boldsymbol{b}}\left(\boldsymbol{x} ; \boldsymbol{x}_{0}\right):=\frac{1}{2 \pi\left|\boldsymbol{x}-\boldsymbol{x}_{0}\right|^{2}} \boldsymbol{b} \otimes\left(\boldsymbol{x}-\boldsymbol{x}_{0}\right)^{\perp}+\nabla \boldsymbol{v}\left(\boldsymbol{x}-\boldsymbol{x}_{0}\right),
$$

with

$$
\boldsymbol{v}(\boldsymbol{x})=-\frac{\mu \log |\boldsymbol{x}|}{2 \pi(\lambda+2 \mu)} \boldsymbol{b}^{\perp}-\frac{\lambda+\mu}{4 \pi(\lambda+2 \mu)|\boldsymbol{x}|^{2}}\left\{\left(\boldsymbol{b} \cdot \boldsymbol{x}^{\perp}\right) \boldsymbol{x}+(\boldsymbol{b} \cdot \boldsymbol{x}) \boldsymbol{x}^{\perp}\right\},
$$

which is a solution in the distributional sense of the system

$$
\left\{\begin{array}{l}
\operatorname{Curl} \boldsymbol{H}=\boldsymbol{b} \delta_{\boldsymbol{x}_{0}} \\
\operatorname{Div} C[\boldsymbol{E}(\boldsymbol{H})]=0
\end{array} \quad \text { in } \mathbb{R}^{2} .\right.
$$

Proof. By Proposition 2.1 and Remark 2.2 the functions $\boldsymbol{K}_{\boldsymbol{b}, \varepsilon, R}$ are given by the solutions of (2.9) in $B_{R}\left(\boldsymbol{x}_{0}\right) \backslash B_{\varepsilon}\left(\boldsymbol{x}_{0}\right)$ satisfying (3.2). In the isotropic case, these are explicitly known [34]:

$$
\boldsymbol{K}_{\boldsymbol{b}, \varepsilon, R}\left(\boldsymbol{x} ; \boldsymbol{x}_{0}\right)=\boldsymbol{K}_{\boldsymbol{b}}\left(\boldsymbol{x} ; \boldsymbol{x}_{0}\right)+\nabla \boldsymbol{w}_{\boldsymbol{b}, \varepsilon, R}\left(\boldsymbol{x}-\boldsymbol{x}_{0}\right),
$$

where

$$
\begin{aligned}
\boldsymbol{w}_{\boldsymbol{b}, \varepsilon, R}(\boldsymbol{x})= & \frac{(\lambda+\mu)|\boldsymbol{x}|^{2}}{2 \pi(\lambda+2 \mu)\left(\varepsilon^{2}+R^{2}\right)}\left\{-\boldsymbol{b}^{\perp}-\frac{\lambda+3 \mu}{2(\lambda+\mu)|\boldsymbol{x}|^{2}}\left(\left(\boldsymbol{b} \cdot \boldsymbol{x}^{\perp}\right) \boldsymbol{x}+(\boldsymbol{b} \cdot \boldsymbol{x}) \boldsymbol{x}^{\perp}\right)\right\} \\
& +\frac{(\lambda+\mu) \varepsilon^{2} R^{2}}{2 \pi(\lambda+2 \mu)\left(\varepsilon^{2}+R^{2}\right)|\boldsymbol{x}|^{4}}\left\{\left(\boldsymbol{b} \cdot \boldsymbol{x}^{\perp}\right) \boldsymbol{x}+(\boldsymbol{b} \cdot \boldsymbol{x}) \boldsymbol{x}^{\perp}\right\} .
\end{aligned}
$$

A straightforward calculation shows that $\boldsymbol{K}_{\boldsymbol{b}, \varepsilon, R}$ satisfies the constraint (3.2) and the Euler equations (2.10). Uniform convergence to $\boldsymbol{K}_{\boldsymbol{b}}$ is immediate.

It is easy to see that $\frac{1}{2 \pi\left|\boldsymbol{x}-\boldsymbol{x}_{0}\right|^{2}} \boldsymbol{b} \otimes\left(\boldsymbol{x}-\boldsymbol{x}_{0}\right)^{\perp}$ satisfies

$$
\operatorname{Curl} \boldsymbol{H}=\boldsymbol{b} \delta_{\boldsymbol{x}_{0}}
$$

in the sense of distributions, and it is clear that all other solutions have the form $\frac{1}{2 \pi\left|\boldsymbol{x}-\boldsymbol{x}_{0}\right|^{2}} \boldsymbol{b} \otimes(\boldsymbol{x}-$ $\left.\boldsymbol{x}_{0}\right)^{\perp}+\nabla \boldsymbol{v}, \boldsymbol{v}$ a vector field in $W_{\text {loc }}^{1,1}\left(\mathbb{R}^{2} ; \mathbb{R}^{2}\right)$. A straightforward calculation shows that choosing $\boldsymbol{v}$ as in (3.4) we obtain that $\boldsymbol{K}_{\boldsymbol{b}}$ also satisfies

$$
\operatorname{Div} C[\boldsymbol{E}(\boldsymbol{H})]=0
$$

and the proof is complete.

Remark 3.2. (i) The field $\boldsymbol{K}_{\boldsymbol{b}}$ may be regarded as the deformation induced by a dislocation with Burgers vector $\boldsymbol{b}$ in the whole plane. By introducing polar coordinates $(\varrho, \vartheta)$ centered at $\boldsymbol{x}_{0}$, with associated basis $\left(\boldsymbol{e}_{\varrho}, \boldsymbol{e}_{\vartheta}\right)$, we may write

$$
\boldsymbol{K}_{\boldsymbol{b}}=\frac{1}{2 \pi \varrho} \boldsymbol{b} \otimes \boldsymbol{e}_{\vartheta}+\nabla \boldsymbol{v}_{\boldsymbol{b}}
$$

with

$$
\boldsymbol{v}_{\boldsymbol{b}}=-\frac{\mu \log \varrho}{2 \pi(\lambda+2 \mu)} \boldsymbol{b}^{\perp}-\frac{\lambda+\mu}{4 \pi(\lambda+2 \mu)}\left\{\left(\boldsymbol{b} \cdot \boldsymbol{e}_{\vartheta}\right) \boldsymbol{e}_{\varrho}+\left(\boldsymbol{b} \cdot \boldsymbol{e}_{\varrho}\right) \boldsymbol{e}_{\vartheta}\right\} .
$$

\footnotetext{
${ }^{5}$ Given a vector $\boldsymbol{v}$, we denote by $\boldsymbol{v}^{\perp}$ the vector perpendicular to $\boldsymbol{v}$ obtained by rotating $\boldsymbol{v}$ counterclockwise by $\pi / 2$.
} 
The complete expression for $\boldsymbol{K}_{\boldsymbol{b}}$ in polar coordinates is the following

$$
\begin{aligned}
\boldsymbol{K}_{\boldsymbol{b}}= & \frac{1}{2 \pi \varrho(\lambda+2 \mu)}\left[\mu\left(\boldsymbol{b} \cdot \boldsymbol{e}_{\vartheta}\right) \boldsymbol{e}_{\varrho} \otimes \boldsymbol{e}_{\varrho}+(2 \lambda+3 \mu)\left(\boldsymbol{b} \cdot \boldsymbol{e}_{\varrho}\right) \boldsymbol{e}_{\varrho} \otimes \boldsymbol{e}_{\vartheta}\right. \\
& \left.-\mu\left(\boldsymbol{b} \cdot \boldsymbol{e}_{\varrho}\right) \boldsymbol{e}_{\vartheta} \otimes \boldsymbol{e}_{\varrho}+\mu\left(\boldsymbol{b} \cdot \boldsymbol{e}_{\vartheta}\right) \boldsymbol{e}_{\vartheta} \otimes \boldsymbol{e}_{\vartheta}\right],
\end{aligned}
$$

and the corresponding stress tensor is

$$
\begin{aligned}
\boldsymbol{T}_{\boldsymbol{b}}= & \frac{\mu(\lambda+\mu)}{\pi \varrho(\lambda+2 \mu)}\left\{\left(\boldsymbol{b} \cdot \boldsymbol{e}_{\vartheta}\right) \boldsymbol{e}_{\varrho} \otimes \boldsymbol{e}_{\varrho}\right. \\
& \left.+\left(\boldsymbol{b} \cdot \boldsymbol{e}_{\varrho}\right)\left(\boldsymbol{e}_{\varrho} \otimes \boldsymbol{e}_{\vartheta}+\boldsymbol{e}_{\vartheta} \otimes \boldsymbol{e}_{\varrho}\right)+\left(\boldsymbol{b} \cdot \boldsymbol{e}_{\vartheta}\right) \boldsymbol{e}_{\vartheta} \otimes \boldsymbol{e}_{\vartheta}\right\} .
\end{aligned}
$$

Note that $\boldsymbol{K}_{\boldsymbol{b}}$ is homogeneous of degree -1 in $\varrho$ so that we may write

$$
\boldsymbol{K}_{\boldsymbol{b}}\left(\varrho, \vartheta ; \boldsymbol{x}_{0}\right)=\frac{1}{\varrho} \boldsymbol{L}_{\boldsymbol{b}}(\vartheta),
$$

where $\boldsymbol{L}_{\boldsymbol{b}}$ is independent of $\varrho$ and $\boldsymbol{x}_{0}$.

(ii) In what follows we shall use extensively the family of tensor fields

$$
\boldsymbol{K}_{\boldsymbol{b}, \varepsilon}\left(\boldsymbol{x} ; \boldsymbol{x}_{0}\right):=\boldsymbol{K}_{\boldsymbol{b}}\left(\boldsymbol{x} ; \boldsymbol{x}_{0}\right)+\nabla \boldsymbol{w}_{\boldsymbol{b}, \varepsilon}\left(\boldsymbol{x}-\boldsymbol{x}_{0}\right),
$$

with

$$
\boldsymbol{w}_{\boldsymbol{b}, \varepsilon}(\boldsymbol{x})=\lim _{R \rightarrow \infty} \boldsymbol{w}_{\boldsymbol{b}, \varepsilon, R}(\boldsymbol{x})=\frac{(\lambda+\mu) \varepsilon^{2}}{2 \pi(\lambda+2 \mu)|\boldsymbol{x}|^{4}}\left\{\left(\boldsymbol{b} \cdot \boldsymbol{x}^{\perp}\right) \boldsymbol{x}+(\boldsymbol{b} \cdot \boldsymbol{x}) \boldsymbol{x}^{\perp}\right\} .
$$

which have the property that $\operatorname{Div} C\left[\boldsymbol{E}\left(\boldsymbol{K}_{\boldsymbol{b}, \varepsilon}\right)\right]=0$ on $\mathbb{R}^{2} \backslash B_{\varepsilon}\left(\boldsymbol{x}_{0}\right)$, and $C\left[\boldsymbol{E}\left(\boldsymbol{K}_{\boldsymbol{b}, \varepsilon}\right)\right] \boldsymbol{n}=0$ on $\partial B_{\varepsilon}\left(\boldsymbol{x}_{0}\right)$. Notice also that $\boldsymbol{w}_{\boldsymbol{b}, \varepsilon} \rightarrow 0$ uniformly on compacta in $\mathbb{R}^{2} \backslash\left\{\boldsymbol{x}_{0}\right\}$.

\section{Existence for systems of dislocations in a bounded domain}

In this section we study the asymptotic behavior as $\varepsilon \rightarrow 0^{+}$of the solutions of the minimization problem

$$
\left.\min _{\boldsymbol{H} \in H\left(\boldsymbol{b}_{1}, \ldots, \boldsymbol{b}_{N} ; \Omega_{\varepsilon}\right)} \int_{\Omega_{\varepsilon}} W(\boldsymbol{E}(\boldsymbol{H}))\right) d a,
$$

where, we recall,

$$
H\left(\boldsymbol{b}_{1}, \ldots, \boldsymbol{b}_{N} ; \Omega_{\varepsilon}\right)=\left\{\boldsymbol{H} \in H\left(\operatorname{Curl} 0 ; \Omega_{\varepsilon}\right): \int_{\partial B_{\varepsilon}\left(\boldsymbol{x}_{i}\right)} \boldsymbol{H} \boldsymbol{t} d s=\boldsymbol{b}_{i}, \quad i=1, \ldots, N\right\},
$$

and where $\boldsymbol{x}_{1}, \ldots, \boldsymbol{x}_{N}$ and $\boldsymbol{b}_{1}, \ldots, \boldsymbol{b}_{N}$ are given sets of points in $\Omega$ and of Burgers vectors, respectively. The main result of this section is the following

Theorem 4.1. Assume that the elasticity tensor $C$ satisfies condition (2.3). Then the minimization problem

$$
\left.\min _{\boldsymbol{H} \in H\left(\boldsymbol{b}_{1}, \ldots, \boldsymbol{b}_{N} ; \Omega_{\varepsilon}\right)} \int_{\Omega_{\varepsilon}} W(\boldsymbol{E}(\boldsymbol{H}))\right) d a,
$$


admits a unique solution, modulo an infinitesimal rigid-body motion, $\boldsymbol{H}_{\varepsilon}$ which converges as $\varepsilon \rightarrow 0$, strongly in $L_{\mathrm{loc}}^{2}\left(\Omega \backslash \cup_{i=1}^{N}\left\{\boldsymbol{x}_{i}\right\} ; \mathbb{R}^{2 \times 2}\right)$, to a solution, in the distributional sense, of the system

$$
\left\{\begin{array}{l}
\operatorname{Curl} \boldsymbol{H}=\sum_{i=1}^{N} \boldsymbol{b}_{i} \delta_{\boldsymbol{x}_{i}} \\
\operatorname{Div} C[\boldsymbol{E}(\boldsymbol{H})]=0
\end{array}\right.
$$

The proof of the previous theorem is divided in several lemmas. We begin by recalling that any tensor field $\boldsymbol{H} \in H\left(\boldsymbol{b}_{1}, \ldots, \boldsymbol{b}_{N} ; \Omega_{\varepsilon}\right)$ can be written as the sum of a given tensor field in $H\left(\boldsymbol{b}_{1}, \ldots, \boldsymbol{b}_{N} ; \Omega_{\varepsilon}\right)$ and the gradient of a vector field. In particular, we may choose

$$
\boldsymbol{H}=\sum_{i=1}^{N} \boldsymbol{K}_{i, \varepsilon}+\nabla \boldsymbol{u},
$$

with $\boldsymbol{u} \in H^{1}\left(\Omega_{\varepsilon} ; \mathbb{R}^{2}\right)$ and (cf. (3.5))

$$
\boldsymbol{K}_{i, \varepsilon}(\boldsymbol{x}):=\boldsymbol{K}_{\boldsymbol{b}_{i}, \varepsilon}\left(\boldsymbol{x} ; \boldsymbol{x}_{i}\right) .
$$

Notice that $\boldsymbol{K}_{i, \varepsilon}$ satisfies the Euler equations (2.10) on $\mathbb{R}^{2} \backslash B_{\varepsilon}\left(\boldsymbol{x}_{i}\right)$, i.e.,

$$
\begin{cases}\operatorname{Div} C\left[\boldsymbol{E}\left(\boldsymbol{K}_{i, \varepsilon}\right)\right]=0 & \text { in } \mathbb{R}^{2} \backslash B_{\varepsilon}\left(\boldsymbol{x}_{i}\right) \\ C\left[\boldsymbol{E}\left(\boldsymbol{K}_{i, \varepsilon}\right)\right] \boldsymbol{n}=\mathbf{0} & \text { on } \partial B_{\varepsilon}\left(\boldsymbol{x}_{i}\right)\end{cases}
$$

Also, $\int_{\partial B_{\varepsilon}\left(\boldsymbol{x}_{i}\right)} \boldsymbol{K}_{i, \varepsilon} \boldsymbol{t} d s=\boldsymbol{b}_{i}$.

Inserting (4.3) into the energy functional (2.8) and applying the divergence theorem we obtain

$$
J_{\varepsilon}(\boldsymbol{H})=\sum_{i=1}^{N} J_{\varepsilon}\left(\boldsymbol{K}_{i, \varepsilon}\right)+\sum_{i=1}^{N-1} \sum_{j=i+1}^{N} \int_{\Omega_{\varepsilon}} C\left[\boldsymbol{E}\left(\boldsymbol{K}_{i, \varepsilon}\right)\right] \cdot \boldsymbol{K}_{j, \varepsilon} d a+I_{\varepsilon}(\boldsymbol{u}),
$$

with

$$
I_{\varepsilon}(\boldsymbol{u}):=\int_{\Omega_{\varepsilon}} W(\boldsymbol{E}(\boldsymbol{u})) d a+\sum_{i=1}^{N} \int_{\partial \Omega} \boldsymbol{u} \cdot \boldsymbol{T}_{i, \varepsilon} \boldsymbol{n} d s-\sum_{i=1}^{N} \sum_{j \neq i} \int_{\partial B_{\varepsilon}\left(\boldsymbol{x}_{i}\right)} \boldsymbol{u} \cdot \boldsymbol{T}_{j, \varepsilon} \boldsymbol{n} d s,
$$

and where $\boldsymbol{T}_{i, \varepsilon}:=C\left[\boldsymbol{E}\left(\boldsymbol{K}_{i, \varepsilon}\right)\right]$.

Hence, granted the decomposition (4.5), for $\varepsilon$ fixed, the minimization problem (4.1) is equivalent to the problem

$\left(M_{\boldsymbol{u}, \varepsilon}\right)$ : Minimize the functional $I_{\varepsilon}$ over all displacement fields $\boldsymbol{u} \in H^{1}\left(\Omega_{\varepsilon} ; \mathbb{R}^{2}\right)$, i.e., find the solutions of

$$
\min _{\boldsymbol{u} \in H^{1}\left(\Omega_{\varepsilon} ; \mathbb{R}^{2}\right)} I_{\varepsilon}(\boldsymbol{u}) .
$$

Lemma 4.2. The functional $I_{\varepsilon}(\boldsymbol{u})$ is invariant under the rigid transformation $\boldsymbol{u} \mapsto \tilde{\boldsymbol{u}}=\boldsymbol{u}+\boldsymbol{a}+\boldsymbol{b} \times \boldsymbol{x}$.

Proof. Since $\boldsymbol{E}(\tilde{\boldsymbol{u}})=\boldsymbol{E}(\boldsymbol{u})$, from (4.6) we obtain

$$
I_{\varepsilon}(\tilde{\boldsymbol{u}})-I_{\varepsilon}(\boldsymbol{u})=\sum_{i=1}^{N} \int_{\partial \Omega}(\boldsymbol{a}+\boldsymbol{b} \times \boldsymbol{x}) \cdot \boldsymbol{T}_{i, \varepsilon} \boldsymbol{n} d s-\sum_{i=1}^{N} \sum_{j \neq i} \int_{\partial B_{\varepsilon}\left(\boldsymbol{x}_{i}\right)}(\boldsymbol{a}+\boldsymbol{b} \times \boldsymbol{x}) \cdot \boldsymbol{T}_{j, \varepsilon} \boldsymbol{n} d s
$$




$$
\begin{aligned}
= & \boldsymbol{a} \cdot\left(\sum_{i=1}^{N} \int_{\partial \Omega} \boldsymbol{T}_{i, \varepsilon} \boldsymbol{n} d s-\sum_{i=1}^{N} \sum_{j \neq i} \int_{\partial B_{\varepsilon}\left(\boldsymbol{x}_{i}\right)} \boldsymbol{T}_{j, \varepsilon} \boldsymbol{n} d s\right) \\
& +\boldsymbol{b} \cdot\left(\sum_{i=1}^{N} \int_{\partial \Omega} \boldsymbol{x} \times \boldsymbol{T}_{i, \varepsilon} \boldsymbol{n} d s-\sum_{i=1}^{N} \sum_{j \neq i} \int_{\partial B_{\varepsilon}\left(\boldsymbol{x}_{i}\right)} \boldsymbol{x} \times \boldsymbol{T}_{j, \varepsilon} \boldsymbol{n} d s\right) .
\end{aligned}
$$

Now, recalling that $\boldsymbol{T}_{i, \varepsilon} \boldsymbol{n}=\mathbf{0}$ on $\partial B_{\varepsilon}\left(\boldsymbol{x}_{i}\right)$, we may write the expression in the first bracket as

$$
\begin{aligned}
\sum_{i=1}^{N} \int_{\partial \Omega} \boldsymbol{T}_{i, \varepsilon} \boldsymbol{n} d s-\sum_{i=1}^{N} \sum_{j=1}^{N} \int_{\partial B_{\varepsilon}\left(\boldsymbol{x}_{i}\right)} \boldsymbol{T}_{j, \varepsilon} \boldsymbol{n} d s & =\sum_{i=1}^{N}\left(\int_{\partial \Omega} \boldsymbol{T}_{i, \varepsilon} \boldsymbol{n} d s-\sum_{j=1}^{N} \int_{\partial B_{\varepsilon}\left(\boldsymbol{x}_{j}\right)} \boldsymbol{T}_{i, \varepsilon} \boldsymbol{n} d s\right) \\
& =\sum_{i=1}^{N} \int_{\partial \Omega_{\varepsilon}} \boldsymbol{T}_{i, \varepsilon} \boldsymbol{n} d s=\sum_{i=1}^{N} \int_{\Omega_{\varepsilon}} \operatorname{Div} \boldsymbol{T}_{i, \varepsilon} d a,
\end{aligned}
$$

which vanishes since $\operatorname{Div} \boldsymbol{T}_{i, \varepsilon}=0$ in $\Omega_{\varepsilon}$. Analogously, the term in the second bracket of the expression of $I_{\varepsilon}(\tilde{\boldsymbol{u}})-I_{\varepsilon}(\boldsymbol{u})$ may be rewritten as

$$
\sum_{i=1}^{N} \int_{\partial \Omega_{\varepsilon}} \boldsymbol{x} \times \boldsymbol{T}_{i, \varepsilon} \boldsymbol{n} d s=\sum_{i=1}^{N}\left(\int_{\Omega_{\varepsilon}} \boldsymbol{x} \times \operatorname{Div} \boldsymbol{T}_{i, \varepsilon} d a-\int_{\Omega_{\varepsilon}} \operatorname{Axial}\left(\boldsymbol{T}_{i, \varepsilon}\right) d a\right),
$$

which vanishes since $\boldsymbol{T}_{i, \varepsilon}$ is symmetric. In the formula above we have denoted by $\operatorname{Axial}(\boldsymbol{T})$ the axial vector of a tensor $\boldsymbol{T}$, whose cartesian components are $\varepsilon_{a b c} T^{c b}$. We also have used the identity

$$
\partial_{d}\left(\varepsilon_{a b c} x^{b} T^{c d}\right)=\varepsilon_{a b c} x^{b} \partial_{d} T^{c d}+\varepsilon_{a b c} \partial_{d} x^{b} T^{c d}=\varepsilon_{a b c} x^{b} \partial_{d} T^{c d}+\varepsilon_{a b c} T^{c b} .
$$

In view of the previous lemma, it is clear that to minimize the functional $I_{\varepsilon}$ over all displacement fields $\boldsymbol{u} \in H^{1}\left(\Omega_{\varepsilon} ; \mathbb{R}^{2}\right)$ is equivalent to minimize $I_{\varepsilon}$ over all $\boldsymbol{u} \in H^{1}\left(\Omega_{\varepsilon} ; \mathbb{R}^{2}\right)$ such that

$$
\int_{B} \boldsymbol{u} d a=\mathbf{0}, \quad \int_{\Omega_{\varepsilon}}\left(\nabla \boldsymbol{u}-(\nabla \boldsymbol{u})^{\top}\right) d a=0
$$

for a fixed ball $B \subset \Omega_{\varepsilon}$. Conditions (4.8) guarantee the coerciveness of the functional $I_{\varepsilon}$ and in turn the existence of minimizers. Indeed we have the following lemma:

Lemma 4.3. Assume that the elasticity tensor $C$ satisfies condition (2.3). Then there exists two positive constants $K_{1}$ and $K_{2}$ independent of $\varepsilon$ such that

$$
I_{\varepsilon}(\boldsymbol{u}) \geq K_{1}\|\boldsymbol{u}\|_{H^{1}\left(\Omega_{\varepsilon} ; \mathbb{R}^{2}\right)}^{2}-K_{2}\|\boldsymbol{u}\|_{H^{1}\left(\Omega_{\varepsilon} ; \mathbb{R}^{2}\right)},
$$

for every $\boldsymbol{u}_{\varepsilon} \in H^{1}\left(\Omega_{\varepsilon} ; \mathbb{R}^{2}\right)$ satisfying the constraint (4.8). Moreover for every $\varepsilon$ the minimization problem

$$
\min _{\boldsymbol{u} \in H^{1}\left(\Omega_{\varepsilon} ; \mathbb{R}^{2}\right)} I_{\varepsilon}(\boldsymbol{u})
$$

admits a unique solution $\boldsymbol{u}_{\varepsilon} \in H^{1}\left(\Omega_{\varepsilon} ; \mathbb{R}^{2}\right)$ satisfying (4.8) and such that

$$
\left\|\boldsymbol{u}_{\varepsilon}\right\|_{H^{1}\left(\Omega_{\varepsilon} ; \mathbb{R}^{2}\right)} \leq M,
$$

for some positive constant $M$ independent of $\varepsilon$. 
Proof. By the positive definiteness of the elasticity tensor $C$, for $\boldsymbol{u} \in H^{1}\left(\Omega_{\varepsilon} ; \mathbb{R}^{2}\right)$,

$$
\begin{aligned}
I_{\varepsilon}(\boldsymbol{u}) \geq & K_{0} \int_{\Omega_{\varepsilon}}|\boldsymbol{E}(\boldsymbol{u})|^{2} d a-\sum_{i=1}^{N} \sup _{\partial \Omega}\left|\boldsymbol{T}_{i, \varepsilon}\right| \int_{\partial \Omega}|\boldsymbol{u}| d s \\
& -\sum_{i=1}^{N} \sum_{j \neq i} \sup _{\partial B_{\varepsilon}\left(\boldsymbol{x}_{i}\right)}\left|\boldsymbol{T}_{j, \varepsilon}\right| \int_{\partial B_{\varepsilon}\left(\boldsymbol{x}_{i}\right)}|\boldsymbol{u}| d s .
\end{aligned}
$$

By Korn's inequality (see Proposition 6.9) there exists a constant $K_{3}$, independent of $\varepsilon$, such that

$$
\int_{\Omega_{\varepsilon}}\left|\boldsymbol{E}\left(\boldsymbol{u}_{\varepsilon}\right)\right|^{2} d a \geq K_{3}\left\|\boldsymbol{u}_{\varepsilon}\right\|_{H^{1}\left(\Omega_{\varepsilon} ; \mathbb{R}^{2}\right)}^{2}
$$

Now, by the trace theorem $(6.38)$

$$
\int_{\partial \Omega}\left|\boldsymbol{u}_{\varepsilon}\right| d s \leq K_{4}\left\|\boldsymbol{u}_{\varepsilon}\right\|_{H^{1}\left(\Omega_{\varepsilon} ; \mathbb{R}^{2}\right)}, \quad \text { and } \quad \int_{\partial B_{\varepsilon}\left(\boldsymbol{x}_{i}\right)}\left|\boldsymbol{u}_{\varepsilon}\right| d s \leq K_{4}\left\|\boldsymbol{u}_{\varepsilon}\right\|_{H^{1}\left(\Omega_{\varepsilon} ; \mathbb{R}^{2}\right)}
$$

with $K_{4}$ independent of $\varepsilon$. Moreover,

$$
\sup _{\partial B_{\varepsilon}\left(\boldsymbol{x}_{i}\right)}\left|\boldsymbol{T}_{j, \varepsilon}\right| \leq K_{5}, \quad j \neq i
$$

with $K_{5}$ independent of $\varepsilon$.

Combining (4.12), (4.13), (4.14) and (4.15) yields (4.9). In turn, since the functional $I_{\varepsilon}$ is convex and $I_{\varepsilon}(\mathbf{0})=0$ the remaining of the proof follows immediately.

We now study the asymptotic behavior of the minimizers $\boldsymbol{u}_{\varepsilon}$.

Lemma 4.4. Assume that the elasticity tensor $C$ satisfies condition (2.3). Let $\boldsymbol{u}_{\varepsilon} \in H^{1}\left(\Omega_{\varepsilon} ; \mathbb{R}^{2}\right)$ be the unique solution of

$$
\min _{\boldsymbol{u} \in H^{1}\left(\Omega_{\varepsilon} ; \mathbb{R}^{2}\right)} I_{\varepsilon}(\boldsymbol{u})
$$

satisfying (4.8). Then as $\varepsilon \rightarrow 0$ the sequence $\left\{\boldsymbol{u}_{\varepsilon}\right\}$ converges strongly in $H_{\text {loc }}^{1}\left(\Omega \backslash \cup_{i=1}^{N}\left\{\boldsymbol{x}_{i}\right\} ; \mathbb{R}^{2}\right)$ to a solution $\boldsymbol{u}_{0}$ of the minimization problem

$$
\min _{\boldsymbol{u} \in H^{1}\left(\Omega ; \mathbb{R}^{2}\right)} I_{0}(\boldsymbol{u}) .
$$

Here

$$
I_{0}(\boldsymbol{u}):=\int_{\Omega} W(\boldsymbol{E}(\boldsymbol{u})) d a+\sum_{i=1}^{N} \int_{\partial \Omega} \boldsymbol{u} \cdot \boldsymbol{T}_{i} \boldsymbol{n} d s,
$$

where $\boldsymbol{T}_{i}:=C\left[\boldsymbol{E}\left(\boldsymbol{K}_{i}\right)\right]$ and

$$
\boldsymbol{K}_{i}(\boldsymbol{x}):=\boldsymbol{K}_{\boldsymbol{b}_{i}}\left(\boldsymbol{x} ; \boldsymbol{x}_{i}\right)
$$

is the fundamental solution defined in (3.3). Moreover,

$$
I_{\varepsilon}\left(\boldsymbol{u}_{\varepsilon}\right) \rightarrow I_{0}\left(\boldsymbol{u}_{0}\right) .
$$


Proof. By Theorem 6.11, we can extend $\boldsymbol{u}_{\varepsilon}$ to $\Omega$ in such a way that

$$
\left\|\boldsymbol{u}_{\varepsilon}\right\|_{H^{1}\left(\Omega ; \mathbb{R}^{2}\right)} \leq C M,
$$

where $M$ is the constant given by (4.10). Hence there exists a subsequence of $\left\{\boldsymbol{u}_{\varepsilon}\right\}$ not relabelled, such that

$$
\boldsymbol{u}_{\varepsilon} \rightarrow \boldsymbol{u}_{0} \quad \text { in } H^{1}\left(\Omega ; \mathbb{R}^{2}\right),
$$

for some $\boldsymbol{u}_{0} \in H^{1}\left(\Omega ; \mathbb{R}^{2}\right)$. By Hölder's inequality,

$$
\left|\int_{\partial B_{\varepsilon}\left(\boldsymbol{x}_{i}\right)} \boldsymbol{u}_{\varepsilon} \cdot \boldsymbol{T}_{j, \varepsilon} \boldsymbol{n} d s\right|^{2} \leq \int_{\partial B_{\varepsilon}\left(\boldsymbol{x}_{i}\right)}\left|\boldsymbol{u}_{\varepsilon}\right|^{2} d s \int_{\partial B_{\varepsilon}\left(\boldsymbol{x}_{i}\right)}\left|\boldsymbol{T}_{j, \varepsilon}\right|^{2} d s \leq \varepsilon K \sup _{B_{\varepsilon}\left(\boldsymbol{x}_{i}\right)}\left|\boldsymbol{T}_{j, \varepsilon}\right|^{2} M^{2},
$$

which vanishes as $\varepsilon \rightarrow 0$, and where we have used Proposition 6.10, (4.10) and the fact that $\boldsymbol{T}_{j, \varepsilon} \rightarrow \boldsymbol{T}_{j}$ uniformly on $B_{\varepsilon}\left(\boldsymbol{x}_{i}\right) . K$ is a positive real constant. Hence

$$
\lim _{\varepsilon \rightarrow 0} \int_{\partial B_{\varepsilon}\left(\boldsymbol{x}_{i}\right)} \boldsymbol{u}_{\varepsilon} \cdot \boldsymbol{T}_{j, \varepsilon} \boldsymbol{n} d s=0
$$

Fix now $\varepsilon_{0}>0$. For $\varepsilon<\varepsilon_{0}$, by $(4.6)$,

$$
I_{\varepsilon}\left(\boldsymbol{u}_{\varepsilon}\right) \geq \int_{\Omega_{\varepsilon_{0}}} W\left(\boldsymbol{E}\left(\boldsymbol{u}_{\varepsilon}\right)\right) d a+\sum_{i=1}^{N} \int_{\partial \Omega} \boldsymbol{u}_{\varepsilon} \cdot \boldsymbol{T}_{i, \varepsilon} \boldsymbol{n} d s-\sum_{i=1}^{N} \sum_{j \neq i} \int_{\partial B_{\varepsilon}\left(\boldsymbol{x}_{i}\right)} \boldsymbol{u}_{\varepsilon} \cdot \boldsymbol{T}_{j, \varepsilon} \boldsymbol{n} d s .
$$

Letting $\varepsilon \rightarrow 0^{+}$, by standard lower semicontinuity results and (4.21), we obtain that

$$
\liminf _{\varepsilon \rightarrow 0^{+}} I_{\varepsilon}\left(\boldsymbol{u}_{\varepsilon}\right) \geq \int_{\Omega_{\varepsilon_{0}}} W\left(\boldsymbol{E}\left(\boldsymbol{u}_{0}\right)\right) d a+\sum_{i=1}^{N} \int_{\partial \Omega} \boldsymbol{u}_{0} \cdot \boldsymbol{T}_{i} \boldsymbol{n} d s .
$$

Letting $\varepsilon_{0} \rightarrow 0^{+}$we get

$$
\liminf _{\varepsilon \rightarrow 0^{+}} I_{\varepsilon}\left(\boldsymbol{u}_{\varepsilon}\right) \geq I_{0}\left(\boldsymbol{u}_{0}\right)
$$

On the other hand, since

$$
I_{\varepsilon}\left(\boldsymbol{u}_{\varepsilon}\right) \leq I_{\varepsilon}\left(\boldsymbol{u}_{0}\right)
$$

we also have that

$$
\limsup _{\varepsilon \rightarrow 0^{+}} I_{\varepsilon}\left(\boldsymbol{u}_{\varepsilon}\right) \leq I_{0}\left(\boldsymbol{u}_{0}\right)
$$

Hence

$$
\lim _{\varepsilon \rightarrow 0^{+}} I_{\varepsilon}\left(\boldsymbol{u}_{\varepsilon}\right)=I_{0}\left(\boldsymbol{u}_{0}\right) .
$$

To prove strong convergence, notice that (4.22) implies that

$$
\lim _{\varepsilon \rightarrow 0^{+}} \int_{\Omega_{\varepsilon}} W\left(\boldsymbol{E}\left(\boldsymbol{u}_{\varepsilon}\right)\right) d a=\int_{\Omega} W\left(\boldsymbol{E}\left(\boldsymbol{u}_{0}\right)\right) d a,
$$

from which we conclude that, as in Evans [16],

$$
\lim _{\varepsilon \rightarrow 0^{+}} \int_{\Omega_{\varepsilon}}\left|\boldsymbol{E}\left(\boldsymbol{u}_{\varepsilon}\right)-\boldsymbol{E}(\boldsymbol{u})\right|^{2} d a=0
$$


and strong convergence of $\boldsymbol{u}_{\varepsilon}$ in $H_{l o c}^{1}\left(\Omega ; \mathbb{R}^{2}\right)$ follows from Korn's inequality.

Now we claim that $\boldsymbol{u}_{0}$ minimizes $I_{0}$. Indeed for any $\boldsymbol{u} \in H^{1}\left(\Omega ; \mathbb{R}^{2}\right)$ we have that

$$
I_{\varepsilon}(\boldsymbol{u}) \geq I_{\varepsilon}\left(\boldsymbol{u}_{\varepsilon}\right)
$$

so that letting $\varepsilon \rightarrow 0^{+}$and using (4.22),

$$
I_{0}(\boldsymbol{u}) \geq I_{0}\left(\boldsymbol{u}_{0}\right)
$$

Next we claim that $\boldsymbol{u}_{0}$ satisfies

$$
\int_{B} \boldsymbol{u}_{0} d a=\mathbf{0}, \quad \int_{\Omega}\left(\nabla \boldsymbol{u}_{0}-\left(\nabla \boldsymbol{u}_{0}\right)^{\top}\right) d a=0
$$

The first constraint follows immediately from (4.8), since $\left\{\boldsymbol{u}_{\varepsilon}\right\}$ converges strongly in $H_{\text {loc }}^{1}\left(\Omega \backslash \cup_{i=1}^{N}\left\{\boldsymbol{x}_{i}\right\} ; \mathbb{R}^{2}\right)$ to $\boldsymbol{u}_{0}$. To prove the second constraint, let $\boldsymbol{S}_{\varepsilon}:=\frac{1}{2}\left(\nabla \boldsymbol{u}_{\varepsilon}-\left(\nabla \boldsymbol{u}_{\varepsilon}\right)^{\top}\right)$ and $\boldsymbol{S}_{0}=\frac{1}{2}\left(\nabla \boldsymbol{u}_{0}-\left(\nabla \boldsymbol{u}_{0}\right)^{\top}\right)$, and notice that $\boldsymbol{S}_{\varepsilon} \rightarrow \boldsymbol{S}_{0}$ strongly in $L_{\text {loc }}^{2}\left(\Omega \backslash \cup_{i=1}^{N}\left\{\boldsymbol{x}_{i}\right\} ; \mathbb{R}^{2 \times 2}\right)$. Now, denote by $\tilde{\boldsymbol{S}}_{\varepsilon}$ the extension of $\boldsymbol{S}_{\varepsilon}$ to zero on $\Omega$. Then, since $\left\|\nabla \boldsymbol{u}_{\varepsilon}\right\|_{L^{2}\left(\Omega_{\varepsilon} ; \mathbb{R}^{2 \times 2}\right)}$ is bounded independently of $\varepsilon$, it follows that the sequence $\left\|\tilde{\boldsymbol{S}}_{\varepsilon}\right\|_{L^{2}\left(\Omega ; \mathbb{R}^{2 \times 2}\right)}$ is bounded, so that $\tilde{\boldsymbol{S}}_{\varepsilon} \rightarrow \tilde{\boldsymbol{S}}_{0}$ in $L^{2}\left(\Omega ; \mathbb{R}^{2 \times 2}\right)$ for some $\tilde{\boldsymbol{S}}_{0} \in L^{2}\left(\Omega ; \mathbb{R}^{2 \times 2}\right)$. Hence, also $\tilde{\boldsymbol{S}}_{\varepsilon} \rightarrow \tilde{\boldsymbol{S}}_{0}$ weakly in $L_{\text {loc }}^{2}\left(\Omega \backslash\left\{\boldsymbol{x}_{0}\right\} ; \mathbb{R}^{2 \times 2}\right)$, and $\tilde{\boldsymbol{S}}_{0}=\boldsymbol{S}_{0}$. By weak convergence,

$$
0=\int_{\Omega_{\varepsilon}} \boldsymbol{S}_{\varepsilon} d a=\int_{\Omega} \tilde{\boldsymbol{S}}_{\varepsilon} d a \rightarrow \int_{\Omega} \boldsymbol{S}_{0} d a
$$

and the claim follows. Since the minimization problem

$$
\min _{\boldsymbol{u} \in H^{1}\left(\Omega ; \mathbb{R}^{2}\right)} I_{0}(\boldsymbol{u}),
$$

admits a unique solution modulo an infinitesimal rigid-body displacement, we conclude that all sequences $\boldsymbol{u}_{\varepsilon}$ converge strongly to $\boldsymbol{u}_{0}$.

We are now ready to conclude the proof of Theorem 4.1.

Proof of Theorem 4.1. Let $\boldsymbol{u}_{\varepsilon} \in H^{1}\left(\Omega_{\varepsilon} ; \mathbb{R}^{2}\right)$ be the unique solution of

$$
\min _{\boldsymbol{u} \in H^{1}\left(\Omega_{\varepsilon} ; \mathbb{R}^{2}\right)} I_{\varepsilon}(\boldsymbol{u})
$$

satisfying (4.8). It suffices to define

$$
\boldsymbol{H}_{\varepsilon}:=\sum_{i=1}^{N} \boldsymbol{K}_{i, \varepsilon}+\nabla \boldsymbol{u}_{\varepsilon} .
$$

Since, by Proposition 3.1, $\boldsymbol{K}_{i, \varepsilon} \rightarrow \boldsymbol{K}_{i}$ uniformly on compact subsets of $\mathbb{R}^{2} \backslash\left\{\boldsymbol{x}_{i}\right\}$ the proof is concluded. 


\section{The renormalized energy}

In this section we prove a sharp estimate for the minimum energy

$$
\left.\min _{\boldsymbol{H} \in H\left(\boldsymbol{b}_{1}, \ldots, \boldsymbol{b}_{N} ; \Omega_{\varepsilon}\right)} \int_{\Omega_{\varepsilon}} W(\boldsymbol{E}(\boldsymbol{H}))\right) d a,
$$

as the core radius $\varepsilon \rightarrow 0$, and compute the renormalized energy which, being a function of the defect position only, allows to study the equilibrium configurations of the defects and the force acting on them.

Let $\Omega$ a bounded domain with the cone property as before, let $\left\{\boldsymbol{x}_{i}\right\}$ be a system of dislocations in $\Omega$, and let

$$
\bar{R}:=\frac{1}{4} \min \left(\left\{\left|\boldsymbol{x}_{i}-\boldsymbol{x}_{j}\right|: i, j=1, \ldots, N, i \neq j\right\} \cup\left\{\operatorname{dist}\left(\left\{\boldsymbol{x}_{1}, \ldots, \boldsymbol{x}_{N}\right\}, \partial \Omega\right)\right\}\right) .
$$

The main result of this section is the following

Theorem 5.1. Assume that the elasticity tensor $C$ satisfies condition (2.3). Let $\boldsymbol{H}_{\varepsilon} \in H\left(\boldsymbol{b}_{1}, \cdots, \boldsymbol{b}_{N} ; \Omega_{\varepsilon}\right)$ be a solution of

$$
\left.\min _{\boldsymbol{H} \in H\left(\boldsymbol{b}_{1}, \ldots, \boldsymbol{b}_{N} ; \Omega_{\varepsilon}\right)} \int_{\Omega_{\varepsilon}} W(\boldsymbol{E}(\boldsymbol{H}))\right) d a,
$$

for a system of dislocations with Burgers vectors $\boldsymbol{b}_{i}$. Then

$$
\left.\int_{\Omega_{\varepsilon}} W\left(\boldsymbol{E}\left(\boldsymbol{H}_{\varepsilon}\right)\right)\right) d a=\sum_{i=1}^{N} \frac{\mu(\lambda+\mu)}{4 \pi(\lambda+2 \mu)}\left|\boldsymbol{b}_{i}\right|^{2} \ln \frac{1}{\varepsilon}+F\left(\boldsymbol{x}_{1}, \ldots, \boldsymbol{x}_{N}\right)+c+O(\varepsilon),
$$

where

$$
F\left(\boldsymbol{x}_{1}, \ldots, \boldsymbol{x}_{N}\right)=F_{\text {self }}\left(\boldsymbol{x}_{1}, \ldots, \boldsymbol{x}_{N}\right)+F_{\text {int }}\left(\boldsymbol{x}_{1}, \ldots, \boldsymbol{x}_{N}\right)+F_{\text {elastic }}\left(\boldsymbol{x}_{1}, \ldots, \boldsymbol{x}_{N}\right),
$$

is the renormalized energy, with

$$
\left\{\begin{array}{l}
F_{\text {self }}\left(\boldsymbol{x}_{1}, \ldots, \boldsymbol{x}_{N}\right)=\sum_{i=1}^{N} \int_{\Omega \backslash B_{R}\left(\boldsymbol{x}_{i}\right)} W\left(\boldsymbol{E}\left(\boldsymbol{K}_{i}\right)\right) d a+\sum_{i=1}^{N} \frac{\mu(\lambda+\mu)}{4 \pi(\lambda+2 \mu)}\left|\boldsymbol{b}_{i}\right|^{2} \ln R, \\
F_{\mathrm{int}}\left(\boldsymbol{x}_{1}, \ldots, \boldsymbol{x}_{N}\right)=\sum_{i=1}^{N-1} \sum_{j=i+1}^{N} \int_{\Omega} C\left[\boldsymbol{E}\left(\boldsymbol{K}_{i}\right)\right] \cdot \boldsymbol{E}\left(\boldsymbol{K}_{j}\right) d a, \\
F_{\text {elastic }}\left(\boldsymbol{x}_{1}, \ldots, \boldsymbol{x}_{N}\right)=\int_{\Omega} W\left(\boldsymbol{E}\left(\boldsymbol{u}_{0}\right)\right) d a+\sum_{i=1}^{N} \int_{\partial \Omega} \boldsymbol{u}_{0} \cdot \boldsymbol{T}_{i} \boldsymbol{n} d s,
\end{array}\right.
$$

where $\boldsymbol{K}_{i}(\boldsymbol{x}):=\boldsymbol{K}_{\boldsymbol{b}_{i}}\left(\boldsymbol{x} ; \boldsymbol{x}_{i}\right)$ is the fundamental solution defined in (3.3), the function $\boldsymbol{u}_{0}$ is defined in Lemma $4.4, c$ is a constant independent of $\boldsymbol{x}_{1}, \ldots, \boldsymbol{x}_{N}$ and $0<R<\bar{R}$ is arbitrary.

Proof. Consider the fundamental solution $\boldsymbol{K}_{i}(\boldsymbol{x}):=\boldsymbol{K}_{\boldsymbol{b}_{i}}\left(\boldsymbol{x} ; \boldsymbol{x}_{i}\right)$ defined (3.3): by (3.10) we may write

$$
\boldsymbol{K}_{i}\left(\varrho_{i}, \vartheta_{i}\right)=\frac{1}{\varrho_{i}} \boldsymbol{L}_{i}\left(\vartheta_{i}\right)
$$

where $\left(\varrho_{i}, \vartheta_{i}\right)$ are polar coordinates centered at $\boldsymbol{x}_{i}$, and $\boldsymbol{L}_{i}\left(\vartheta_{i}\right)$ is independent of $\varrho_{i}$ and is defined by (3.10), with $\boldsymbol{b}$ replaced by $\boldsymbol{b}_{i}$. A straightforward computation using (3.8) and (3.9) yields

$$
a_{i}:=\int_{0}^{2 \pi} W\left(\boldsymbol{E}\left(\boldsymbol{L}_{i}(\vartheta)\right)\right) d \vartheta=\frac{\mu(\lambda+\mu)}{4 \pi(\lambda+2 \mu)}\left|\boldsymbol{b}_{i}\right|^{2} .
$$


By (4.5) we can write the minimum energy in the form

$$
J_{\varepsilon}\left(\boldsymbol{H}_{\varepsilon}\right)=I_{\varepsilon}\left(\boldsymbol{u}_{\varepsilon}\right)+\sum_{i=1}^{N} J_{\varepsilon}\left(\boldsymbol{K}_{i, \varepsilon}\right)+\sum_{i=1}^{N-1} \sum_{j=i+1}^{N} \int_{\Omega_{\varepsilon}} C\left[\boldsymbol{E}\left(\boldsymbol{K}_{i, \varepsilon}\right)\right] \cdot \boldsymbol{K}_{j, \varepsilon} d a .
$$

where $I_{\varepsilon}\left(\boldsymbol{u}_{\varepsilon}\right)$ is the functional defined by (4.6).

Notice first that the representation of the elastic contribution $F_{\text {elastic }}$ in $(5.3)_{3}$ follows immediately from (4.22).

We now compute the self-energy contribution $F_{\text {self }}$ : fix $R<\bar{R}$ and write

$$
J_{\varepsilon}\left(\boldsymbol{K}_{i, \varepsilon}\right)=\int_{\Omega_{\varepsilon} \backslash B_{R}\left(\boldsymbol{x}_{i}\right)} W\left(\boldsymbol{E}\left(\boldsymbol{K}_{i, \varepsilon}\right)\right) d a+\int_{C_{\varepsilon, R}\left(\boldsymbol{x}_{i}\right)} W\left(\boldsymbol{E}\left(\boldsymbol{K}_{i, \varepsilon}\right)\right) d a,
$$

with $C_{\varepsilon, R}\left(\boldsymbol{x}_{i}\right)=B_{R}\left(\boldsymbol{x}_{i}\right) \backslash B_{\varepsilon}\left(\boldsymbol{x}_{i}\right)$.

Now, as $\varepsilon \rightarrow 0$, by uniform-on-compacta convergence of $\boldsymbol{K}_{i, \varepsilon}$ on $\mathbb{R}^{2} \backslash\left\{\boldsymbol{x}_{i}\right\}$,

$$
\int_{\Omega_{\varepsilon} \backslash B_{R}\left(\boldsymbol{x}_{i}\right)} W\left(\boldsymbol{E}\left(\boldsymbol{K}_{i, \varepsilon}\right)\right) d a \rightarrow \int_{\Omega \backslash B_{R}\left(\boldsymbol{x}_{i}\right)} W\left(\boldsymbol{E}\left(\boldsymbol{K}_{i}\right)\right) d a .
$$

Moreover, writing as in (3.11) $\boldsymbol{K}_{i, \varepsilon}=\boldsymbol{K}_{i}+\nabla \boldsymbol{w}_{\varepsilon}$, with $\boldsymbol{w}_{\varepsilon} \rightarrow 0$ uniformly on compacta in $\mathbb{R}^{2} \backslash\left\{\boldsymbol{x}_{i}\right\}$, we have

$$
\begin{aligned}
\int_{C_{\varepsilon, R}\left(\boldsymbol{x}_{i}\right)} W\left(\boldsymbol{E}\left(\boldsymbol{K}_{i, \varepsilon}\right)\right) d a= & \int_{C_{\varepsilon, R}\left(\boldsymbol{x}_{i}\right)} W\left(\boldsymbol{E}\left(\boldsymbol{K}_{i}\right)\right) d a+\int_{C_{\varepsilon, R}\left(\boldsymbol{x}_{i}\right)} C\left[\boldsymbol{E}\left(\boldsymbol{K}_{i}\right)\right] \cdot \nabla \boldsymbol{w}_{\varepsilon} d a \\
& +\int_{C_{\varepsilon, R}\left(\boldsymbol{x}_{i}\right)} W\left(\boldsymbol{E}\left(\boldsymbol{w}_{\varepsilon}\right)\right) d a
\end{aligned}
$$

and, by (5.4), the first integral on the right hand side of this identity gives

$$
\int_{C_{\varepsilon, R}\left(\boldsymbol{x}_{i}\right)} W\left(\boldsymbol{E}\left(\boldsymbol{K}_{i}\right)\right) d a=a_{i}(\ln R-\ln \varepsilon),
$$

while the second and third integral may be written as

$$
\begin{gathered}
\int_{\partial B_{R}\left(\boldsymbol{x}_{i}\right)} \boldsymbol{w}_{\varepsilon} \cdot C\left[\boldsymbol{E}\left(\boldsymbol{K}_{i}\right)+\frac{1}{2} \boldsymbol{E}\left(\boldsymbol{w}_{\varepsilon}\right)\right] \boldsymbol{n} d s-\int_{\partial B_{\varepsilon}\left(\boldsymbol{x}_{i}\right)} \boldsymbol{w}_{\varepsilon} \cdot C\left[\boldsymbol{E}\left(\boldsymbol{K}_{i}\right)+\frac{1}{2} \boldsymbol{E}\left(\boldsymbol{w}_{\varepsilon}\right)\right] \boldsymbol{n} d s \\
=\int_{\partial B_{R}\left(\boldsymbol{x}_{i}\right)} \boldsymbol{w}_{\varepsilon} \cdot C\left[\boldsymbol{E}\left(\boldsymbol{K}_{i}\right)+\frac{1}{2} \boldsymbol{E}\left(\boldsymbol{w}_{\varepsilon}\right)\right] \boldsymbol{n} d s-\frac{1}{2} \int_{\partial B_{\varepsilon}\left(\boldsymbol{x}_{i}\right)} \boldsymbol{w}_{\varepsilon} \cdot C\left[\boldsymbol{E}\left(\boldsymbol{K}_{i}\right)\right] \boldsymbol{n} d s
\end{gathered}
$$

where we have used the fact that $C\left[\boldsymbol{E}\left(\boldsymbol{w}_{\varepsilon}\right)\right] \boldsymbol{n}=-C\left[\boldsymbol{E}\left(\boldsymbol{K}_{i}\right)\right] \boldsymbol{n}$ on $\partial B_{\varepsilon}\left(\boldsymbol{x}_{i}\right)$, since $C\left[\boldsymbol{E}\left(\boldsymbol{K}_{i, \varepsilon}\right)\right] \boldsymbol{n}=\mathbf{0}$ on $\partial B_{\varepsilon}\left(\boldsymbol{x}_{i}\right)$. The first integral on the right hand side of the above expression vanishes as $\varepsilon \rightarrow 0$, while by (3.12) we may write

$$
\boldsymbol{w}_{\varepsilon}\left(\varrho_{i}, \vartheta_{i}\right)=\frac{\varepsilon^{2}}{\varrho_{i}^{2}} \overline{\boldsymbol{w}}\left(\vartheta_{i}\right),
$$

which, in conjunction with (5.4), shows that

$$
-\frac{1}{2} \int_{\partial B_{\varepsilon}\left(\boldsymbol{x}_{i}\right)} \boldsymbol{w}_{\varepsilon} \cdot C\left[\boldsymbol{E}\left(\boldsymbol{K}_{i}\right)\right] \boldsymbol{n} d s=-\frac{1}{2} \int_{0}^{2 \pi} \overline{\boldsymbol{w}}(\vartheta) \cdot C\left[\boldsymbol{E}\left(\boldsymbol{L}_{i}(\vartheta)\right)\right] \boldsymbol{n} d \vartheta=c,
$$


with $c$ a constant independent of $\left(\boldsymbol{x}_{1}, \ldots, \boldsymbol{x}_{N}\right)$. To summarize, (5.10) converges, as $\varepsilon \rightarrow 0$, to a constant independent of $R$ and $\boldsymbol{x}_{i}$.

Notice that $F_{\text {self }}$ is independent of $R$, since, for $R^{\prime}<\bar{R}$, say $R^{\prime}<R$,

$$
\begin{aligned}
\int_{\Omega \backslash B_{R^{\prime}}\left(\boldsymbol{x}_{i}\right)} W\left(\boldsymbol{E}\left(\boldsymbol{K}_{i}\right)\right) d a+a_{i} \ln R^{\prime} & =\int_{\Omega \backslash B_{R}\left(\boldsymbol{x}_{i}\right)} W\left(\boldsymbol{E}\left(\boldsymbol{K}_{i}\right)\right) d a+\int_{C_{R^{\prime}, R}\left(\boldsymbol{x}_{i}\right)} W\left(\boldsymbol{E}\left(\boldsymbol{K}_{i}\right)\right) d a+a_{i} \ln R^{\prime} \\
\quad= & \int_{\Omega \backslash B_{R}\left(\boldsymbol{x}_{i}\right)} W\left(\boldsymbol{E}\left(\boldsymbol{K}_{i}\right)\right) d a+a_{i} \ln \frac{R}{R^{\prime}}+a_{i} \ln R^{\prime} \\
= & \int_{\Omega \backslash B_{R}\left(\boldsymbol{x}_{i}\right)} W\left(\boldsymbol{E}\left(\boldsymbol{K}_{i}\right)\right) d a+a_{i} \ln R .
\end{aligned}
$$

We finally compute the contribution of the interaction term $F_{\text {int }}$ to the renormalized energy, and prove that

$$
\int_{\Omega_{\varepsilon}} C\left[\boldsymbol{E}\left(\boldsymbol{K}_{i, \varepsilon}\right)\right] \cdot \boldsymbol{E}\left(\boldsymbol{K}_{j, \varepsilon}\right) d a=\int_{\Omega} C\left[\boldsymbol{E}\left(\boldsymbol{K}_{i}\right)\right] \cdot \boldsymbol{E}\left(\boldsymbol{K}_{j}\right) d a+O(\varepsilon) .
$$

To see this, let as before $\boldsymbol{K}_{i, \varepsilon}=\boldsymbol{K}_{i}+\nabla \boldsymbol{w}_{i, \varepsilon}$ and $\boldsymbol{K}_{j, \varepsilon}=\boldsymbol{K}_{j}+\nabla \boldsymbol{w}_{j, \varepsilon}$ (cf. (3.11)), so that

$$
\begin{aligned}
\int_{\Omega_{\varepsilon}} C\left[\boldsymbol{E}\left(\boldsymbol{K}_{i, \varepsilon}\right)\right] \cdot \boldsymbol{E}\left(\boldsymbol{K}_{j, \varepsilon}\right) d a= & \int_{\Omega_{\varepsilon}} C\left[\boldsymbol{E}\left(\boldsymbol{K}_{i}\right)\right] \cdot \boldsymbol{E}\left(\boldsymbol{K}_{j}\right) d a+\int_{\Omega_{\varepsilon}} C\left[\boldsymbol{E}\left(\boldsymbol{K}_{i}\right)\right] \cdot \nabla \boldsymbol{w}_{j, \varepsilon} d a \\
& +\int_{\Omega_{\varepsilon}} C\left[\boldsymbol{E}\left(\boldsymbol{K}_{j}\right)\right] \cdot \nabla \boldsymbol{w}_{i, \varepsilon} d a+\int_{\Omega_{\varepsilon}} C\left[\boldsymbol{E}\left(\boldsymbol{w}_{i, \varepsilon}\right)\right] \cdot \nabla \boldsymbol{w}_{j, \varepsilon} d a .
\end{aligned}
$$

It can be easily proved that

$$
\int_{\Omega_{\varepsilon}} C\left[\boldsymbol{E}\left(\boldsymbol{K}_{i}\right)\right] \cdot \boldsymbol{E}\left(\boldsymbol{K}_{j}\right) d a \rightarrow \int_{\Omega} C\left[\boldsymbol{E}\left(\boldsymbol{K}_{i}\right)\right] \cdot \boldsymbol{E}\left(\boldsymbol{K}_{j}\right) d a,
$$

while, applying the divergence theorem, the last three integrals become

$$
\begin{aligned}
& \int_{\partial \Omega}\left(\boldsymbol{w}_{j, \varepsilon} \cdot C\left[\boldsymbol{E}\left(\boldsymbol{K}_{i}\right)\right] \boldsymbol{n}+\boldsymbol{w}_{i, \varepsilon} \cdot C\left[\boldsymbol{E}\left(\boldsymbol{K}_{j}\right)\right] \boldsymbol{n}+\boldsymbol{w}_{j, \varepsilon} \cdot C\left[\boldsymbol{E}\left(\boldsymbol{w}_{i, \varepsilon}\right)\right] \boldsymbol{n}\right) d a \\
& \quad-\sum_{k=1}^{N} \int_{\partial B_{\varepsilon}\left(\boldsymbol{x}_{k}\right)}\left(\boldsymbol{w}_{j, \varepsilon} \cdot C\left[\boldsymbol{E}\left(\boldsymbol{K}_{i}\right)\right] \boldsymbol{n}+\boldsymbol{w}_{i, \varepsilon} \cdot C\left[\boldsymbol{E}\left(\boldsymbol{K}_{j}\right)\right] \boldsymbol{n}+\boldsymbol{w}_{j, \varepsilon} \cdot C\left[\boldsymbol{E}\left(\boldsymbol{w}_{i, \varepsilon}\right)\right] \boldsymbol{n}\right) d a .
\end{aligned}
$$

Now, recall that $\boldsymbol{w}_{i, \varepsilon} \rightarrow 0$ uniformly on compacta in $\mathbb{R}^{2} \backslash\left\{\boldsymbol{x}_{i}\right\}$ (cf. (3.5)), so that the integrals over $\partial \Omega$ and $\partial B_{\varepsilon}\left(\boldsymbol{x}_{k}\right)$, with $k \neq i, j$ vanish in the limit as $\varepsilon \rightarrow 0$, and (5.12) becomes

$$
\begin{aligned}
& -\int_{\partial B_{\varepsilon}\left(\boldsymbol{x}_{i}\right)}\left(\boldsymbol{w}_{j, \varepsilon} \cdot C\left[\boldsymbol{E}\left(\boldsymbol{K}_{i}\right)\right] \boldsymbol{n}+\boldsymbol{w}_{i, \varepsilon} \cdot C\left[\boldsymbol{E}\left(\boldsymbol{K}_{j}\right)\right] \boldsymbol{n}+\boldsymbol{w}_{j, \varepsilon} \cdot C\left[\boldsymbol{E}\left(\boldsymbol{w}_{i, \varepsilon}\right)\right] \boldsymbol{n}\right) d a \\
& -\int_{\partial B_{\varepsilon}\left(\boldsymbol{x}_{j}\right)}\left(\boldsymbol{w}_{j, \varepsilon} \cdot C\left[\boldsymbol{E}\left(\boldsymbol{K}_{i}\right)\right] \boldsymbol{n}+\boldsymbol{w}_{i, \varepsilon} \cdot C\left[\boldsymbol{E}\left(\boldsymbol{K}_{j}\right)\right] \boldsymbol{n}+\boldsymbol{w}_{j, \varepsilon} \cdot C\left[\boldsymbol{E}\left(\boldsymbol{w}_{i, \varepsilon}\right)\right] \boldsymbol{n}\right) d a,
\end{aligned}
$$

which tends to 0 as $\varepsilon \rightarrow 0$. Consider in fact the first term: then

$$
\left|\int_{\partial B_{\varepsilon}\left(\boldsymbol{x}_{i}\right)} \boldsymbol{w}_{j, \varepsilon} \cdot C\left[\boldsymbol{E}\left(\boldsymbol{K}_{i}\right)\right] \boldsymbol{n} d a\right| \leq C \sup _{B_{\varepsilon}\left(\boldsymbol{x}_{i}\right)}\left|\boldsymbol{w}_{j, \varepsilon}\right| \int_{\partial B_{\varepsilon}\left(\boldsymbol{x}_{i}\right)}\left|C\left[\boldsymbol{E}\left(\boldsymbol{K}_{i}\right)\right] \boldsymbol{n}\right| d a
$$




$$
\leq \sup _{B_{\varepsilon}\left(\boldsymbol{x}_{i}\right)}\left|\boldsymbol{w}_{j, \varepsilon}\right| \int_{\partial B_{\varepsilon}\left(\boldsymbol{x}_{i}\right)}\left|\boldsymbol{K}_{i}\right| d a \rightarrow 0
$$

by uniform convergence of $\boldsymbol{w}_{j, \varepsilon}$. Consider now the second term in (5.13): as before, we may write

$$
\boldsymbol{w}_{i, \varepsilon}\left(\varrho_{i}, \vartheta_{i}\right)=\frac{\varepsilon^{2}}{\varrho_{i}^{2}} \overline{\boldsymbol{w}}_{i}\left(\vartheta_{i}\right)
$$

so that, since $\boldsymbol{K}_{j}$ is continuous at $\boldsymbol{x}_{i}$,

$$
\left|\int_{\partial B_{\varepsilon}\left(\boldsymbol{x}_{i}\right)} \boldsymbol{w}_{i, \varepsilon} \cdot C\left[\boldsymbol{E}\left(\boldsymbol{K}_{j}\right)\right] \boldsymbol{n} d s\right| \leq \sup _{B_{\varepsilon}\left(\boldsymbol{x}_{i}\right)}\left|C\left[\boldsymbol{E}\left(\boldsymbol{K}_{j}\right)\right]\right| \int_{\partial B_{\varepsilon}\left(\boldsymbol{x}_{i}\right)}\left|\overline{\boldsymbol{w}}_{i}\right| d s \rightarrow 0
$$

as $\varepsilon \rightarrow 0$, since $\overline{\boldsymbol{w}}_{i}$ is bounded. The remaining terms in (5.13) can be treated analogously, and this completes the proof of (5.1).

Proposition 5.2. The interaction energy $F_{\text {int }}$ in $(5.3)_{2}$ diverges logarithmically with the relative distance between the defects:

$$
F_{\mathrm{int}}\left(\boldsymbol{x}_{1}, \ldots, \boldsymbol{x}_{N}\right)=\sum_{i=1}^{N-1} \sum_{j=i+1}^{N} \frac{\mu(\lambda+\mu)}{\pi(\lambda+2 \mu)} \boldsymbol{b}_{i} \cdot \boldsymbol{b}_{j} \ln \frac{1}{\left|\boldsymbol{x}_{i}-\boldsymbol{x}_{j}\right|}+O(1),
$$

as $\left|\boldsymbol{x}_{i}-\boldsymbol{x}_{j}\right| \rightarrow 0$.

Proof. Recall that $\boldsymbol{K}_{i} \in L^{1}\left(\Omega ; \mathbb{R}^{2 \times 2}\right)$ for each $i$, and

$$
F_{\mathrm{int}}\left(\boldsymbol{x}_{1}, \ldots, \boldsymbol{x}_{N}\right)=\sum_{i=1}^{N-1} \sum_{j=i+1}^{N} \int_{\Omega} C\left[\boldsymbol{E}\left(\boldsymbol{K}_{i}\right)\right] \cdot \boldsymbol{E}\left(\boldsymbol{K}_{j}\right) d a,
$$

let $\boldsymbol{x}_{i}, \boldsymbol{x}_{j} \in \Omega$, and $\gamma$ a line segment parallel to $\boldsymbol{x}_{j}-\boldsymbol{x}_{i}$ connecting $\boldsymbol{x}_{j}$ to $\partial \Omega$ (cf. Figure 1), so that $\gamma=\left\{\boldsymbol{x} \in \Omega: \boldsymbol{x}=\boldsymbol{x}_{j}+s\left(\boldsymbol{x}_{j}-\boldsymbol{x}_{i}\right)\right\}$, with $s \in[0, \bar{s}]$. Let moreover $\boldsymbol{m}=\left(\frac{\boldsymbol{x}_{j}-\boldsymbol{x}_{i}}{\left|\boldsymbol{x}_{j}-\boldsymbol{x}_{i}\right|}\right)^{\perp}$ be the unit vector orthogonal to $\gamma$. Consider the tensor field $\boldsymbol{K}_{j}$ : while $\Omega \backslash\left\{\boldsymbol{x}_{j}\right\}$ is not simply connected, $\Omega \backslash \gamma$ is, so that there exists a field $\boldsymbol{w}_{j}$ on $\Omega \backslash \gamma$ such that $\boldsymbol{K}_{j}=\nabla \boldsymbol{w}_{j}$, and

$$
\llbracket \boldsymbol{w}_{j} \rrbracket=-\boldsymbol{b}_{j},
$$

where $\llbracket \boldsymbol{w}_{j} \rrbracket$ is the jump of $\boldsymbol{w}_{j}$ across $\gamma$, defined by

$$
\llbracket \boldsymbol{w}_{j} \rrbracket(\boldsymbol{x}):=\lim _{\boldsymbol{y} \rightarrow \boldsymbol{x}, \boldsymbol{y} \cdot \boldsymbol{m}>0} \boldsymbol{w}_{j}(\boldsymbol{y})-\lim _{\boldsymbol{y} \rightarrow \boldsymbol{x}, \boldsymbol{y} \cdot \boldsymbol{m}<0} \boldsymbol{w}_{j}(\boldsymbol{y})
$$

for $\boldsymbol{x} \in \gamma$. Applying the divergence theorem to $\Omega \backslash \gamma$, and noting that $\partial(\Omega \backslash \gamma)=\partial \Omega \cup \gamma$, we find

$$
\begin{aligned}
\int_{\Omega} C\left[\boldsymbol{E}\left(\boldsymbol{K}_{i}\right)\right] \cdot \boldsymbol{K}_{j} d a & =\int_{\Omega \backslash \gamma} C\left[\boldsymbol{E}\left(\boldsymbol{K}_{i}\right)\right] \cdot \nabla \boldsymbol{w}_{j} d a \\
& =\int_{\partial \Omega} \boldsymbol{w}_{j} \cdot C\left[\boldsymbol{E}\left(\boldsymbol{K}_{i}\right)\right] \boldsymbol{n} d s-\int_{\gamma} \llbracket \boldsymbol{w}_{j} \rrbracket \cdot C\left[\boldsymbol{E}\left(\boldsymbol{K}_{i}\right)\right] \boldsymbol{m} d s .
\end{aligned}
$$


The first integral in the above expression remains bounded as $\boldsymbol{x}_{i}-\boldsymbol{x}_{j} \rightarrow \mathbf{0}$, since $\boldsymbol{K}_{i}(\boldsymbol{x})=\boldsymbol{K}_{\boldsymbol{b}_{i}}\left(\boldsymbol{x} ; \boldsymbol{x}_{i}\right) \rightarrow$ $\boldsymbol{K}_{\boldsymbol{b}_{i}}\left(\boldsymbol{x} ; \boldsymbol{x}_{j}\right)$ uniformly on $\partial \Omega$ as $\boldsymbol{x}_{j}-\boldsymbol{x}_{j} \rightarrow \mathbf{0}$. As to the second integral, write $\boldsymbol{K}_{i}=\frac{1}{\varrho_{i}} \boldsymbol{L}_{i}\left(\vartheta_{i}\right)$, and choose $s=\varrho_{i}-d, \vartheta_{i}=\bar{\vartheta}$ on $\gamma$ with $d=\left|\boldsymbol{x}_{j}-\boldsymbol{x}_{i}\right|$ : then

$$
\begin{aligned}
-\int_{\gamma} \llbracket \boldsymbol{w}_{j} \rrbracket \cdot C\left[\boldsymbol{E}\left(\boldsymbol{K}_{i}\right)\right] \boldsymbol{m} d s & =\int_{0}^{\bar{s}} \boldsymbol{b}_{j} \cdot C\left[\boldsymbol{E}\left(\boldsymbol{L}_{i}(\bar{\vartheta})\right)\right] \boldsymbol{m} \frac{1}{d+s} d s \\
& =\boldsymbol{b}_{j} \cdot C\left[\boldsymbol{E}\left(\boldsymbol{L}_{i}(\bar{\vartheta})\right)\right] \boldsymbol{m}\left(\ln \frac{1}{d}+\ln (d+\bar{s})\right)
\end{aligned}
$$

which proves (5.14) since by a straightforward computation using (3.8) and (3.9) we have

$$
\boldsymbol{b}_{j} \cdot C\left[\boldsymbol{E}\left(\boldsymbol{L}_{i}(\bar{\vartheta})\right)\right] \boldsymbol{m}=\frac{\mu(\lambda+\mu)}{\pi(\lambda+2 \mu)} \boldsymbol{b}_{i} \cdot \boldsymbol{b}_{j}
$$

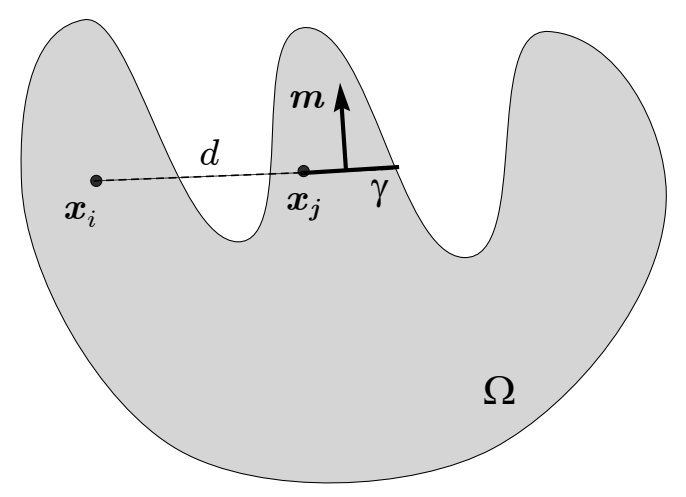

Figure 1: Connecting cut $\gamma$.

\section{The force on a dislocation}

We prove in this section that the derivative of the renormalized energy with respect to defect position coincides with the resultant of the Eshelby stress

$$
\boldsymbol{C}=W(\boldsymbol{E}(\boldsymbol{H})) \mathbf{1}-\boldsymbol{H}^{\top} C[\boldsymbol{E}(\boldsymbol{H})]
$$

on the dislocation.

In order to highlight the dependence of the minimizers on the location of the dislocations, we write

$$
\boldsymbol{u}_{0}\left(\boldsymbol{x} ; \boldsymbol{x}_{1}, \ldots, \boldsymbol{x}_{N}\right)
$$


for a minimizer of $I_{0}$ relative to a system of dislocations located at $\left(\boldsymbol{x}_{1}, \ldots, \boldsymbol{x}_{N}\right)$ in $\Omega$, and

$$
\boldsymbol{H}_{0}=\boldsymbol{H}_{0}\left(\boldsymbol{x} ; \boldsymbol{x}_{1}, \ldots, \boldsymbol{x}_{N}\right):=\sum_{i=1}^{N} \boldsymbol{K}_{\boldsymbol{b}_{i}}\left(\boldsymbol{x} ; \boldsymbol{x}_{i}\right)+\nabla \boldsymbol{u}_{0}\left(\boldsymbol{x} ; \boldsymbol{x}_{1}, \ldots, \boldsymbol{x}_{N}\right)
$$

for the corresponding solution of (4.2) as in Theorem 2.1. Let also $\boldsymbol{h} \in \mathbb{R}^{2}$ be a fixed vector and $t \in I \subset \mathbb{R}$ a real parameter.

Lemma 6.1. The field $\boldsymbol{K}_{\boldsymbol{b}_{i}}\left(\boldsymbol{x} ; \boldsymbol{x}_{i}\right), \boldsymbol{u}_{0}\left(\boldsymbol{x} ; \boldsymbol{x}_{1}, \ldots, \boldsymbol{x}_{N}\right)$ and $\boldsymbol{H}_{0}\left(\boldsymbol{x} ; \boldsymbol{x}_{1}, \ldots, \boldsymbol{x}_{N}\right)$ are smooth with respect to $\boldsymbol{x}_{i}$ for $i \in\{1, \ldots, N\}$.

In particular,

$$
\dot{\boldsymbol{K}}_{i}(\boldsymbol{x}):=\left.\frac{d}{d t} \boldsymbol{K}_{\boldsymbol{b}_{i}}\left(\boldsymbol{x} ; \boldsymbol{x}_{i}+t \boldsymbol{h}\right)\right|_{t=0}=-\nabla\left(\boldsymbol{K}_{\boldsymbol{b}_{i}}\left(\boldsymbol{x} ; \boldsymbol{x}_{i}\right) \boldsymbol{h}\right)=-\nabla\left(\boldsymbol{K}_{i}(\boldsymbol{x}) \boldsymbol{h}\right) .
$$

Moreover, if for a fixed $k \in\{1, \ldots, N\}$ we denote by $\dot{\boldsymbol{u}}_{0}$ and $\dot{\boldsymbol{H}}_{0}$ the smooth fields such that

$$
\begin{aligned}
\dot{\boldsymbol{u}}_{0}(\boldsymbol{x}) & :=\left.\frac{d}{d t} \boldsymbol{u}_{0}\left(\boldsymbol{x} ; \boldsymbol{x}_{1}, \ldots, \boldsymbol{x}_{k}+t \boldsymbol{h}, \ldots, \boldsymbol{x}_{N}\right)\right|_{t=0}, \\
\dot{\boldsymbol{H}}_{0}(\boldsymbol{x}) & :=\left.\frac{d}{d t} \boldsymbol{H}_{0}\left(\boldsymbol{x} ; \boldsymbol{x}_{1}, \ldots, \boldsymbol{x}_{k}+t \boldsymbol{h}, \ldots, \boldsymbol{x}_{N}\right)\right|_{t=0},
\end{aligned}
$$

then

$$
\dot{\boldsymbol{H}}_{0}=\nabla \boldsymbol{w}, \quad \text { with } \quad \boldsymbol{w}=\dot{\boldsymbol{u}}_{0}-\boldsymbol{K}_{k} \boldsymbol{h} .
$$

Proof. Smoothness of $\boldsymbol{K}_{i}$ follows upon recalling that $\boldsymbol{K}_{\boldsymbol{b}_{i}}\left(\boldsymbol{x} ; \boldsymbol{x}_{i}\right)$ is a smooth function of $\boldsymbol{x}-\boldsymbol{x}_{i}$ (cf. (3.3)). This implies that $\boldsymbol{K}_{\boldsymbol{b}_{i}}\left(\boldsymbol{x} ; \boldsymbol{x}_{i}+t \boldsymbol{h}\right)=\boldsymbol{K}_{\boldsymbol{b}_{i}}\left(\boldsymbol{x}-t \boldsymbol{h} ; \boldsymbol{x}_{i}\right)=\boldsymbol{K}_{i}(\boldsymbol{x}-t \boldsymbol{h})$, so that

$$
\dot{\boldsymbol{K}}_{i}(\boldsymbol{x})=-\nabla\left(\boldsymbol{K}_{i}(\boldsymbol{x})\right) \boldsymbol{h},
$$

where $\nabla\left(\boldsymbol{K}_{i}\right) \boldsymbol{h}$ is the tensor field defined by the identity $\left[\nabla\left(\boldsymbol{K}_{i}\right) \boldsymbol{h}\right] \boldsymbol{z}=\left[\nabla\left(\boldsymbol{K}_{i} \boldsymbol{z}\right)\right] \boldsymbol{h}$ for any constant vector $\boldsymbol{z} \in \mathbb{R}^{2}$. Since Curl $\boldsymbol{K}_{i}=0$ in $\mathbb{R}^{2} \backslash\left\{\boldsymbol{x}_{i}\right\}$,

$$
\nabla\left(\boldsymbol{K}_{i}\right) \boldsymbol{h}=\nabla\left(\boldsymbol{K}_{i} \boldsymbol{h}\right)
$$

which yields (6.3)

Now, since $\boldsymbol{u}_{0}$ minimizes the functional $I_{0}$, and satisfies the corresponding Euler equations, it may be written in the form

$$
\boldsymbol{u}_{0}\left(\boldsymbol{x} ; \boldsymbol{x}_{1}, \ldots, \boldsymbol{x}_{N}\right)=\int_{\partial \Omega} \boldsymbol{G}(\boldsymbol{x}, \boldsymbol{\xi})\left(\sum_{i=1}^{N} \boldsymbol{T}_{i}\left(\boldsymbol{\xi} ; \boldsymbol{x}_{1}, \ldots, \boldsymbol{x}_{N}\right) \boldsymbol{n}(\boldsymbol{\xi})\right) d a_{\boldsymbol{\xi}},
$$

modulo an infinitesimal rigid body motion, where $\boldsymbol{G}(\boldsymbol{x}, \boldsymbol{\xi})$ is the Green's function for the Neumann problem in plane elasticity. Since

$$
\sum_{i=1}^{N} \boldsymbol{T}_{i}\left(\boldsymbol{x} ; \boldsymbol{x}_{1}, \ldots, \boldsymbol{x}_{N}\right)=\sum_{i=1}^{N} C\left[\boldsymbol{E}\left(\boldsymbol{K}_{\boldsymbol{b}_{i}}\left(\boldsymbol{x} ; \boldsymbol{x}_{i}\right)\right)\right]
$$

the smoothness of $\boldsymbol{u}_{o}$ follows from the smoothness of $\boldsymbol{K}_{i}$.

Finally, the smoothness of $\boldsymbol{H}_{0}$ follows from (6.2), and (6.4) can be directly verified. 
Lemma 6.2. Let $f=f(\boldsymbol{x}, t), g=g(\boldsymbol{x}, t), r=r(\boldsymbol{x}, t)$, be smooth functions defined on $B_{R}\left(\boldsymbol{x}_{0}+t \boldsymbol{h}\right)$, $\partial B_{R}\left(\boldsymbol{x}_{0}+\right.$ th $)$ and $\Omega \backslash B_{R}\left(\boldsymbol{x}_{0}+\right.$ th $)$ for $t \in I \subset \mathbb{R}$ respectively, with $R>0$ and $\boldsymbol{h}$ a constant vector. Then

$$
\begin{aligned}
\left.\frac{d}{d t} \int_{B_{R}\left(\boldsymbol{x}_{0}+t \boldsymbol{h}\right)} f(\boldsymbol{x}, t) d a\right|_{t=0} & =\int_{B_{R}\left(\boldsymbol{x}_{0}\right)} D_{t} f(\boldsymbol{x}, 0) d a \\
=\int_{B_{R}\left(\boldsymbol{x}_{0}\right)} \partial_{t} f(\boldsymbol{x}, 0) d a & +\int_{\partial B_{R}\left(\boldsymbol{x}_{0}\right)} f(\boldsymbol{x}, 0) \boldsymbol{h} \cdot \boldsymbol{n} d s
\end{aligned}
$$

and

$$
\left.\frac{d}{d t} \int_{\partial B_{R}\left(\boldsymbol{x}_{0}+t \boldsymbol{h}\right)} g(\boldsymbol{x}, t) d s\right|_{t=0}=\int_{\partial B_{R}\left(\boldsymbol{x}_{0}\right)} D_{t} g(\boldsymbol{x}, 0) d s
$$

Moreover,

$$
\left.\frac{d}{d t} \int_{\Omega \backslash B_{R}\left(\boldsymbol{x}_{0}+t \boldsymbol{h}\right)} r(\boldsymbol{x}, t) d a\right|_{t=0}=\int_{\Omega \backslash B_{R}\left(\boldsymbol{x}_{0}\right)} \partial_{t} r(\boldsymbol{x}, 0) d a-\int_{\partial B_{R}\left(\boldsymbol{x}_{0}\right)} r(\boldsymbol{x}, 0) \boldsymbol{h} \cdot \boldsymbol{n} d s
$$

where

$$
D_{t} f=\partial_{t} f+\nabla f \cdot \boldsymbol{h}
$$

Proof. The first identity follows upon applying the classical theorem of derivation under the integral

$$
\begin{aligned}
& \frac{d}{d t} \int_{B_{R}\left(\boldsymbol{x}_{0}+t \boldsymbol{h}\right)} f(\boldsymbol{x}, t) d a=\frac{d}{d t} \int_{B_{R}\left(\boldsymbol{x}_{0}\right)} f(\boldsymbol{x}+t \boldsymbol{h}, t) d a \\
& =\int_{B_{R}\left(\boldsymbol{x}_{0}\right)}\left(\partial_{t} f(\boldsymbol{x}+t \boldsymbol{h}, t)+\boldsymbol{h} \cdot \nabla f(\boldsymbol{x}+t \boldsymbol{h}, t)\right) d a
\end{aligned}
$$

letting $t=0$ and using the divergence theorem we obtain (6.6). Relation (6.7) follows from a similar argument. To prove (6.8), denote by $\hat{r}(\boldsymbol{x}, t)$ a smooth extension of $r(\boldsymbol{x}, t)$ to $\Omega$ for all $t \in I$. Then

$$
\begin{aligned}
\frac{d}{d t} \int_{\Omega \backslash B_{R}\left(\boldsymbol{x}_{0}+t \boldsymbol{h}\right)} r(\boldsymbol{x}, t) d a= & \frac{d}{d t} \int_{\Omega} \hat{r}(\boldsymbol{x}, t) d a-\frac{d}{d t} \int_{B_{R}\left(\boldsymbol{x}_{0}+t \boldsymbol{h}\right)} \hat{r}(\boldsymbol{x}, t) d a \\
= & \int_{\Omega} \partial_{t} \hat{r}(\boldsymbol{x}, t) d a-\int_{B_{R}\left(\boldsymbol{x}_{0}+t \boldsymbol{h}\right)} \partial_{t} \hat{r}(\boldsymbol{x}, t) d a \\
& -\int_{\partial B_{R}\left(\boldsymbol{x}_{0}+t \boldsymbol{h}\right)} \hat{r}(\boldsymbol{x}, t) \boldsymbol{h} \cdot \boldsymbol{n} d s \\
= & \int_{\Omega \backslash B_{R}\left(\boldsymbol{x}_{0}+t \boldsymbol{h}\right)} \partial_{t} \hat{r}(\boldsymbol{x}, t) d a-\int_{\partial B_{R}\left(\boldsymbol{x}_{0}+t \boldsymbol{h}\right)} \hat{r}(\boldsymbol{x}, t) \boldsymbol{h} \cdot \boldsymbol{n} d s,
\end{aligned}
$$

which proves the assertion.

The next theorem, one of our main results, shows that the derivative of the renormalized energy coincides with the force on a dislocation and thus, as mentioned in the introduction, it may be viewed as the generalization of Eshelby's notion of force on a defect, when bad singularities are associated to the defect itself. 
Theorem 6.3. Let $\boldsymbol{H}_{0}$ be defined by (6.2), and let $F=F\left(\boldsymbol{x}_{1}, \ldots, \boldsymbol{x}_{N}\right)$ be the renormalized energy (5.2): then

$$
\nabla_{\boldsymbol{x}_{k}} F=-\int_{\partial B_{R}\left(\boldsymbol{x}_{k}\right)}\left\{W\left(\boldsymbol{E}\left(\boldsymbol{H}_{0}\right)\right) \mathbf{1}-\boldsymbol{H}_{0}^{\top} C\left[\boldsymbol{E}\left(\boldsymbol{H}_{0}\right)\right]\right\} \boldsymbol{n} d s
$$

for $R<\frac{1}{2} \min \left(\left\{\left|\boldsymbol{x}_{i}-\boldsymbol{x}_{j}\right|: i, j=1, \ldots, N, i \neq j\right\} \cup\left\{\operatorname{dist}\left(\left\{\boldsymbol{x}_{1}, \ldots, \boldsymbol{x}_{N}\right\}, \partial \Omega\right)\right\}\right)$.

Proof. The first step is to rewrite the renormalized energy as the sum of two contributions:

$$
F\left(\boldsymbol{x}_{1}, \ldots, \boldsymbol{x}_{N}\right)=F_{R}\left(\boldsymbol{x}_{1}, \ldots, \boldsymbol{x}_{N}\right)+G\left(\boldsymbol{x}_{1}, \ldots, \boldsymbol{x}_{N}\right),
$$

where we have omitted the constant logarithmic term and

$$
F_{R}\left(\boldsymbol{x}_{1}, \ldots, \boldsymbol{x}_{N}\right)=\int_{\Omega_{R}} W\left(\boldsymbol{E}\left(\boldsymbol{H}_{0}\right)\right) d a,
$$

and

$$
\begin{aligned}
G\left(\boldsymbol{x}_{1}, \ldots, \boldsymbol{x}_{N}\right)= & \sum_{i=1}^{N} \sum_{h \neq i} \int_{B_{R}\left(\boldsymbol{x}_{h}\right)} W\left(\boldsymbol{E}\left(\boldsymbol{K}_{i}\right)\right) d a+\sum_{h=1}^{N} \sum_{i=1}^{N-1} \sum_{j=i+1}^{N} \int_{B_{R}\left(\boldsymbol{x}_{h}\right)} C\left[\boldsymbol{E}\left(\boldsymbol{K}_{i}\right)\right] \cdot \boldsymbol{K}_{j} d a \\
& +\sum_{h=1}^{N} \int_{B_{R}\left(\boldsymbol{x}_{h}\right)} W\left(\boldsymbol{E}\left(\boldsymbol{u}_{0}\right)\right) d a+\sum_{h=1}^{N} \sum_{i=1}^{N} \int_{\partial B_{R}\left(\boldsymbol{x}_{h}\right)} \boldsymbol{u}_{0} \cdot C\left[\boldsymbol{E}\left(\boldsymbol{K}_{i}\right)\right] \boldsymbol{n} d a .
\end{aligned}
$$

Consider now a variation of the position of the $k$-th dislocation of the form $\boldsymbol{x}_{k} \rightarrow \boldsymbol{x}_{k}+t \boldsymbol{h}$, with $\boldsymbol{h}$ a fixed vector. Then

$$
\begin{aligned}
& \left.\frac{d}{d t} F_{R}\left(\boldsymbol{x}_{1}, \ldots, \boldsymbol{x}_{k}+t \boldsymbol{h}, \ldots, \boldsymbol{x}_{N}\right)\right|_{t=0} \\
& \quad=\left.\frac{d}{d t} \int_{\Omega_{R} \backslash\left(B_{R}\left(\boldsymbol{x}_{1}\right) \cup \cdots \cup B_{R}\left(\boldsymbol{x}_{k}+t \boldsymbol{h}\right) \cup \cdots \cup B_{R}\left(\boldsymbol{x}_{N}\right)\right)} W\left(\boldsymbol{E}\left(\boldsymbol{H}_{0}\left(\boldsymbol{x} ; \boldsymbol{x}_{1}, \ldots, \boldsymbol{x}_{k}+t \boldsymbol{h}, \ldots, \boldsymbol{x}_{N}\right)\right)\right) d a\right|_{t=0}
\end{aligned}
$$

and applying Lemma 6.2 we obtain

$$
\int_{\Omega_{R}} C\left[\boldsymbol{E}\left(\boldsymbol{H}_{0}\right)\right] \cdot \dot{\boldsymbol{H}}_{0} d a-\int_{\partial B_{R}\left(\boldsymbol{x}_{k}\right)} W\left(\boldsymbol{E}\left(\boldsymbol{H}_{0}\right)\right) \boldsymbol{h} \cdot \boldsymbol{n} d s .
$$

Recalling now Lemma 6.1 , writing $\dot{\boldsymbol{H}}_{0}=\nabla \boldsymbol{w}$, applying the divergence theorem and recalling that $C\left[\boldsymbol{E}\left(\boldsymbol{H}_{0}\right)\right] \boldsymbol{n}=0$ on $\partial \Omega$ this becomes

$$
\begin{array}{rl}
\int_{\Omega_{R}} & C\left[\boldsymbol{E}\left(\boldsymbol{H}_{0}\right)\right] \cdot \nabla \boldsymbol{w} d a-\int_{\partial B_{R}\left(\boldsymbol{x}_{k}\right)} W\left(\boldsymbol{E}\left(\boldsymbol{H}_{0}\right)\right) \boldsymbol{h} \cdot \boldsymbol{n} d s \\
= & -\sum_{i=1}^{N} \int_{\partial B_{R}\left(\boldsymbol{x}_{i}\right)} \boldsymbol{w} \cdot C\left[\boldsymbol{E}\left(\boldsymbol{H}_{0}\right)\right] \boldsymbol{n} d s-\int_{\partial B_{R}\left(\boldsymbol{x}_{k}\right)} W\left(\boldsymbol{E}\left(\boldsymbol{H}_{0}\right)\right) \boldsymbol{h} \cdot \boldsymbol{n} d s
\end{array}
$$

which in turn equals

$$
-\int_{\partial B_{R}\left(\boldsymbol{x}_{k}\right)} \boldsymbol{h} \cdot\left(W\left(\boldsymbol{E}\left(\boldsymbol{H}_{0}\right)\right) \mathbf{1}-\boldsymbol{H}_{0}^{\top} C\left[\boldsymbol{E}\left(\boldsymbol{H}_{0}\right)\right]\right) \boldsymbol{n} d s
$$




$$
-\int_{\partial B_{R}\left(\boldsymbol{x}_{k}\right)}\left(\boldsymbol{w}+\boldsymbol{H}_{0} \boldsymbol{h}\right) \cdot C\left[\boldsymbol{E}\left(\boldsymbol{H}_{0}\right)\right] \boldsymbol{n} d s-\sum_{i \neq k} \int_{\partial B_{R}\left(\boldsymbol{x}_{i}\right)} \boldsymbol{w} \cdot C\left[\boldsymbol{E}\left(\boldsymbol{H}_{0}\right)\right] \boldsymbol{n} d s .
$$

The first term is the resultant of the Eshelby stress on the $k$-th dislocation, while the second and third term may be written as

$$
\begin{gathered}
-\int_{\partial B_{R}\left(\boldsymbol{x}_{k}\right)}\left(\boldsymbol{u}_{0}+\left(\nabla \boldsymbol{u}_{0}\right) \boldsymbol{h}+\sum_{i \neq k} \boldsymbol{K}_{i} \boldsymbol{h}\right) \cdot C\left[\boldsymbol{E}\left(\boldsymbol{H}_{0}\right)\right] \boldsymbol{n} d s \\
-\sum_{i \neq k} \int_{\partial B_{R}\left(\boldsymbol{x}_{i}\right)}\left(\dot{\boldsymbol{u}}_{0}-\boldsymbol{K}_{k} \boldsymbol{h}\right) \cdot C\left[\boldsymbol{E}\left(\boldsymbol{H}_{0}\right)\right] \boldsymbol{n} d s .
\end{gathered}
$$

since $\boldsymbol{w}=\boldsymbol{u}_{0}-\boldsymbol{K}_{k} \boldsymbol{h}$ and

$$
\boldsymbol{w}+\boldsymbol{H}_{0} \boldsymbol{h}=\dot{\boldsymbol{u}_{0}}-\boldsymbol{K}_{k} \boldsymbol{h}+\left(\sum_{i} \boldsymbol{K}_{i}+\nabla \boldsymbol{u}_{0}\right) \boldsymbol{h}=\boldsymbol{u}_{0}+\left(\nabla \boldsymbol{u}_{0}\right) \boldsymbol{h}+\sum_{i \neq k} \boldsymbol{K}_{i} \boldsymbol{h} .
$$

We now prove that (6.14) cancels with the derivative of (6.13), i.e.,

$$
\left.\frac{d}{d t} G\left(\boldsymbol{x}_{1}, \ldots, \boldsymbol{x}_{k}+t \boldsymbol{h}, \ldots, \boldsymbol{x}_{N}\right)\right|_{t=0} .
$$

In fact, rewriting (6.13) as the sum of the terms

$$
\begin{aligned}
& \int_{B_{R}\left(\boldsymbol{x}_{k}\right)}\left\{\sum_{i \neq k} W\left(\boldsymbol{E}\left(\boldsymbol{K}_{i}\right)\right)+\sum_{i<j} C\left[\boldsymbol{E}\left(\boldsymbol{K}_{i}\right)\right] \cdot \boldsymbol{K}_{j}+W\left(\boldsymbol{E}\left(\boldsymbol{u}_{0}\right)\right)\right\} d a \\
& +\int_{\partial B_{R}\left(\boldsymbol{x}_{k}\right)} \sum_{i}\left(\boldsymbol{u}_{0} \cdot C\left[\boldsymbol{E}\left(\boldsymbol{K}_{i}\right)\right] \boldsymbol{n}\right) d s
\end{aligned}
$$

and

$$
\begin{aligned}
& \sum_{h \neq k} \int_{B_{R}\left(\boldsymbol{x}_{h}\right)}\left\{\sum_{i \neq h} W\left(\boldsymbol{E}\left(\boldsymbol{K}_{i}\right)\right)+\sum_{i<j} C\left[\boldsymbol{E}\left(\boldsymbol{K}_{i}\right)\right] \cdot \boldsymbol{K}_{j}+W\left(\boldsymbol{E}\left(\boldsymbol{u}_{0}\right)\right)\right\} d a \\
& \quad+\sum_{h \neq k} \int_{\partial B_{R}\left(\boldsymbol{x}_{h}\right)} \sum_{i}\left(\boldsymbol{u}_{0} \cdot C\left[\boldsymbol{E}\left(\boldsymbol{K}_{i}\right)\right] \boldsymbol{n}\right) d s,
\end{aligned}
$$

and computing the derivative of (6.15) with respect to $t$ and using Lemma 6.2, we find

$$
\begin{aligned}
& \int_{B_{R}\left(\boldsymbol{x}_{k}\right)}\left\{\sum_{i \neq k} C\left[\boldsymbol{E}\left(\boldsymbol{K}_{i}\right)\right] \cdot D_{t} \boldsymbol{K}_{i}+\sum_{i<j} C\left[\boldsymbol{E}\left(\boldsymbol{K}_{i}\right)\right] \cdot D_{t} \boldsymbol{K}_{j}\right. \\
& \left.+\sum_{i<j} C\left[\boldsymbol{E}\left(\boldsymbol{K}_{j}\right)\right] \cdot D_{t} \boldsymbol{K}_{i}+C\left[\boldsymbol{E}\left(\boldsymbol{u}_{0}\right)\right] \cdot \nabla\left(D_{t} \boldsymbol{u}_{0}\right)\right\} d a \\
& +\int_{\partial B_{R}\left(\boldsymbol{x}_{k}\right)}\left\{\sum_{i} \boldsymbol{u}_{0} \cdot C\left[\boldsymbol{E}\left(D_{t} \boldsymbol{K}_{i}\right)\right] \boldsymbol{n}+\sum_{i} D_{t} \boldsymbol{u}_{0} \cdot C\left[\boldsymbol{E}\left(\boldsymbol{K}_{i}\right)\right] \boldsymbol{n}\right\} d s
\end{aligned}
$$


where $D_{t} \boldsymbol{K}_{j}:=\dot{\boldsymbol{K}}_{j}+\nabla\left(\boldsymbol{K}_{j}\right) \boldsymbol{h}$ and $D_{t} \boldsymbol{u}_{0}=\dot{\boldsymbol{u}}_{0}+\left(\nabla \boldsymbol{u}_{0}\right) \boldsymbol{h}$. Since $D_{t} \boldsymbol{K}_{k}=0$ and $D_{t} \boldsymbol{K}_{j}=\nabla\left(\boldsymbol{K}_{j}\right) \boldsymbol{h}=$ $\nabla\left(\boldsymbol{K}_{j} \boldsymbol{h}\right)$ for $j \neq k$ (since $\dot{\boldsymbol{K}}_{j}=0$ for $j \neq k$ ), using the divergence theorem and noting that $\operatorname{Div} C\left[\boldsymbol{E}\left(D_{t} \boldsymbol{K}_{i}\right)\right]=$ 0 on $\mathbb{R}^{2} \backslash\left\{\boldsymbol{x}_{i}\right\}$, we find

$$
\begin{gathered}
\int_{\partial B_{R}\left(\boldsymbol{x}_{k}\right)}\left\{D_{t} \boldsymbol{u}_{0} \cdot C\left[\boldsymbol{E}\left(\boldsymbol{H}_{0}\right)\right] \boldsymbol{n}+\sum_{i \neq k}\left(\boldsymbol{K}_{i} \boldsymbol{h}\right) \cdot C\left[\boldsymbol{E}\left(\boldsymbol{u}_{0}\right)\right] \boldsymbol{n}\right. \\
\left.+\sum_{i \neq k}\left(\boldsymbol{K}_{i} \boldsymbol{h}\right) \cdot C\left[\boldsymbol{E}\left(\boldsymbol{K}_{i}\right)\right] \boldsymbol{n}+\sum_{i \neq k} \sum_{j \neq i}\left(\boldsymbol{K}_{i} \boldsymbol{h}\right) \cdot C\left[\boldsymbol{E}\left(\boldsymbol{K}_{j}\right)\right] \boldsymbol{n}\right\} d s
\end{gathered}
$$

which becomes finally

$$
\int_{\partial B_{R}\left(\boldsymbol{x}_{k}\right)}\left(D_{t} \boldsymbol{u}_{0}+\sum_{i \neq k} \boldsymbol{K}_{i} \boldsymbol{h}\right) \cdot C\left[\boldsymbol{E}\left(\boldsymbol{H}_{0}\right)\right] \boldsymbol{n} d s .
$$

Consider now the derivative of (6.16) with respect to $t$ : using Lemma 6.2, we find

$$
\begin{aligned}
& \sum_{h \neq k} \int_{B_{R}\left(\boldsymbol{x}_{h}\right)}\left\{C\left[\boldsymbol{E}\left(\boldsymbol{K}_{k}\right)\right] \cdot \dot{\boldsymbol{K}}_{k}+\sum_{i \neq k} C\left[\boldsymbol{E}\left(\boldsymbol{K}_{i}\right)\right] \cdot \dot{\boldsymbol{K}}_{k}+C\left[\boldsymbol{E}\left(\boldsymbol{u}_{0}\right)\right] \cdot \nabla \dot{\boldsymbol{u}}_{0}\right\} d a \\
& \quad+\sum_{h \neq k} \int_{\partial B_{R}\left(\boldsymbol{x}_{h}\right)}\left\{\boldsymbol{u}_{0} \cdot C\left[\boldsymbol{E}\left(\dot{\boldsymbol{K}}_{k}\right)\right] \boldsymbol{n}+\sum_{i} \dot{\boldsymbol{u}}_{0} \cdot C\left[\boldsymbol{E}\left(\boldsymbol{K}_{i}\right)\right] \boldsymbol{n}\right\} d s,
\end{aligned}
$$

and using the fact that $\operatorname{Div} C\left[\boldsymbol{E}\left(\dot{\boldsymbol{K}}_{k}\right)\right]=0$ on $\mathbb{R}^{2} \backslash\left\{\boldsymbol{x}_{k}\right\}$, and $\dot{\boldsymbol{K}}_{k}=-\nabla\left(\boldsymbol{K}_{k} \boldsymbol{h}\right)$ we finally obtain

$$
\sum_{h \neq k} \int_{\partial B_{R}\left(\boldsymbol{x}_{h}\right)}\left(\dot{\boldsymbol{u}}_{0}-\boldsymbol{K}_{k} \boldsymbol{h}\right) \cdot C\left[\boldsymbol{E}\left(\boldsymbol{H}_{0}\right)\right] \boldsymbol{n} d s .
$$

Relation (6.10) follows now upon noting that the sum of (6.14), (6.17) and (6.18) vanishes.

\section{Appendix: Poincaré and Korn inequalities for $\Omega_{\varepsilon}$}

The basic tool to study the compactness of a sequence of minimizers of problem (2.9) is Korn's inequality: we prove here that, for a perforated domain such as $\Omega_{\varepsilon}$, under mild regularity assumptions, Korn's and Poincaré's inequalities hold uniformly in $\varepsilon$ as $\varepsilon \rightarrow 0$. Also, we show that the trace constant for $\Omega_{\varepsilon}$ may be chosen independent of $\varepsilon$.

The proof of the following proposition, which follows closely that of [10], provides an upper bound of Poincaré's constant in terms of the decomposition of $\Omega$ into star-shaped domains $A$ (cf. (6.24)-(6.25)). We believe that this result is of interest in itself. For simplicity we consider only plane domains, but the proof goes through in any dimension with obvious modifications.

We recall that an open set $\Omega \subset \mathbb{R}^{2}$ is starshaped with respect to a set $S \subset \Omega$ if $\Omega$ is starshaped with respect to each point of $S$, i.e. if $\boldsymbol{x} \in \Omega$ and $s \in S$ then $\vartheta \boldsymbol{x}+(1-\vartheta) s \in \Omega$ for all $\vartheta \in(0,1)$. 
Proposition 6.4. Let $\Omega \subset \mathbb{R}^{2}$ be an open bounded domain having the cone property. Let $\boldsymbol{u} \in H^{1}\left(\Omega ; \mathbb{R}^{2}\right)$. Then

$$
\int_{\Omega}\left|\boldsymbol{u}-\boldsymbol{u}_{B}\right|^{2} d a \leq C \int_{\Omega}|\nabla \boldsymbol{u}|^{2} d a
$$

where

$$
\boldsymbol{u}_{B}:=\frac{1}{|B|} \int_{B} \boldsymbol{u} d a,
$$

$B$ is any fixed ball whose closure is contained in $\Omega$, and $C$ is a positive constant depending only on $\Omega$ and on the ball $B$.

Proof. Since $\Omega$ has the cone property, it is the union of a finite number of domains starshaped with respect to a ball. Let $d$ be a number greater than the diameter of all these domains, and let $A$ be any of these subdomains with $D$ being the corresponding ball. Construct a finite family of balls $B_{0}, \cdots, B_{M}$ contained in $\Omega$ and such that $B_{0}=D, B_{i} \cap B_{i+1} \neq \emptyset, B_{M}=B$. Since $A$ is starshaped with respect to any fixed ball $\tilde{B}$ contained in $B_{0} \cap B_{1}$, by Lemma 7.16 in [22] we obtain

$$
\int_{A} \frac{\left|\boldsymbol{u}-\boldsymbol{u}_{\tilde{B}}\right|^{2}}{d^{2}} d a \leq\left(\frac{\pi d^{2}}{|A|}\right)^{\frac{1}{2}} \frac{|A|}{|\tilde{B}|} \int_{A}|\nabla \boldsymbol{u}|^{2} d a
$$

where $d$ is any number greater or equal than the diameter of $\Omega$. Using the inequality $(a+b)^{2} \leq 2 a^{2}+2 a^{2}$ we obtain

$$
\begin{aligned}
\int_{A}\left(\frac{|\boldsymbol{u}|}{d}\right)^{2} d a & \leq 2|A|\left(\frac{\left|\boldsymbol{u}_{\tilde{B}}\right|}{d}\right)^{2}+2\left(\frac{\pi d^{2}}{|A|}\right)^{\frac{1}{2}} \frac{|A|}{|\tilde{B}|} \int_{A}|\nabla \boldsymbol{u}|^{2} d a \\
& \leq 2 \frac{|A|}{|\tilde{B}|} \int_{\tilde{B}}\left(\frac{|\boldsymbol{u}|}{d}\right)^{2} d a+2\left(\frac{\pi d^{2}}{|A|}\right)^{\frac{1}{2}} \frac{|A|}{|\tilde{B}|} \int_{A}|\nabla \boldsymbol{u}|^{2} d a
\end{aligned}
$$

where we have used Jensen's inequality. Hence

$$
\int_{A}\left(\frac{|\boldsymbol{u}|}{d}\right)^{2} d a \leq 2 \frac{|A|}{|\tilde{B}|} \int_{B_{0} \cap B_{1}}\left(\frac{|\boldsymbol{u}|}{d}\right)^{2} d a+2\left(\frac{\pi d^{2}}{|A|}\right)^{\frac{1}{2}} \frac{|A|}{|\tilde{B}|} \int_{A}|\nabla \boldsymbol{u}|^{2} d a .
$$

Similarly, since for $i=1, \cdots, M-1$ the ball $B_{i}$ is starshaped with respect to any fixed ball $\tilde{B}_{i}$ contained in $B_{i} \cap B_{i+1} \neq \emptyset$, we obtain

$$
\int_{B_{i}}\left(\frac{|\boldsymbol{u}|}{d}\right)^{2} d a \leq 2 \frac{\left|B_{i}\right|}{\left|\tilde{B}_{i}\right|} \int_{B_{i} \cap B_{i+1}}\left(\frac{|\boldsymbol{u}(\boldsymbol{x})|}{d}\right)^{2} d a+2\left(\frac{\pi d^{2}}{\left|B_{i}\right|}\right)^{\frac{1}{2}} \frac{\left|B_{i}\right|}{\left|\tilde{B}_{i}\right|} \int_{B_{i}}|\nabla \boldsymbol{u}|^{2} d a .
$$

Therefore by (6.21) and (6.22) we get

$$
\int_{A}\left(\frac{|\boldsymbol{u}|}{d}\right)^{2} d a \leq C_{1} \int_{B}\left(\frac{|\boldsymbol{u}|}{d}\right)^{2} d a+C_{2} \int_{\Omega}|\nabla \boldsymbol{u}|^{2} d a,
$$


where

$$
\begin{aligned}
& C_{1}:=2^{M} \frac{|A|}{|\tilde{B}|} \prod_{i=1}^{M} \frac{\left|B_{i}\right|}{\left|\tilde{B}_{i}\right|} \\
& C_{2}:=2\left(\pi d^{2}\right)^{\frac{1}{2}}\left(\frac{1}{|A|^{\frac{1}{2}}} \frac{|A|}{|\tilde{B}|}+2 \frac{1}{\left|B_{1}\right|^{\frac{1}{2}}} \frac{|A|}{|\tilde{B}|} \frac{\left|B_{1}\right|}{\left|\tilde{B}_{1}\right|}+\cdots+2^{M} \frac{1}{\left|B_{M}\right|^{\frac{1}{2}}} \frac{|A|}{|\tilde{B}|} \frac{\left|B_{1}\right|}{\left|\tilde{B}_{1}\right|} \cdots \frac{\left|B_{M}\right|}{\left|\tilde{B}_{M}\right|}\right)
\end{aligned}
$$

Summing over all $A$ gives

$$
\int_{\Omega}\left(\frac{|\boldsymbol{u}|}{d}\right)^{2} d a \leq \# A\left(C_{1} \int_{B}\left(\frac{|\boldsymbol{u}|}{d}\right)^{2} d a+C_{2} \int_{\Omega}|\nabla \boldsymbol{u}|^{2} d a\right) .
$$

Since $B$ is convex, by Lemma 7.16 in [22] (1983) we obtain

$$
\int_{B}\left(\frac{\left|\boldsymbol{u}-\boldsymbol{u}_{B}\right|}{d}\right)^{2} d a \leq\left(\frac{\pi d^{2}}{|B|}\right)^{\frac{1}{2}} \int_{B}|\nabla \boldsymbol{u}|^{2} d a
$$

where $\boldsymbol{u}_{B}:=\frac{1}{|B|} \int_{B} \boldsymbol{u} d a$. Replacing $\boldsymbol{u}$ by $\boldsymbol{u}-\boldsymbol{u}_{B}$ in (6.26) we obtain

$$
\begin{aligned}
\int_{\Omega}\left(\frac{\left|\boldsymbol{u}-\boldsymbol{u}_{B}\right|}{d}\right)^{2} d a & \leq \# A\left(C_{1} \int_{B}\left(\frac{\left|\boldsymbol{u}-\boldsymbol{u}_{B}\right|}{d}\right)^{2}+C_{2} \int_{\Omega}|\nabla \boldsymbol{u}|^{2} d a\right) \\
& \leq \# A\left(C_{1}\left(\frac{\pi d^{2}}{|B|}\right)^{\frac{1}{2}}+C_{2}\right) \int_{\Omega}|\nabla \boldsymbol{u}|^{2} d a
\end{aligned}
$$

that is

$$
\int_{\Omega}\left|\boldsymbol{u}-\boldsymbol{u}_{B}\right|^{2} d a \leq d^{2} \# A\left(C_{1}\left(\frac{\pi d^{2}}{|B|}\right)^{\frac{1}{2}}+C_{2}\right) \int_{\Omega}|\nabla \boldsymbol{u}|^{2} d a .
$$

This concludes the proof.

We now prove that Poincaré's inequality holds for each domain $\Omega_{\varepsilon}$ uniformly in $\varepsilon$, when $\Omega$ has the cone property.

Proposition 6.5. Let $\Omega$ be a bounded open domain in $\mathbb{R}^{2}$ with the cone property: then, for any $\boldsymbol{u} \in$ $H^{1}\left(\Omega_{\varepsilon} ; \mathbb{R}^{2}\right)$,

$$
\int_{\Omega_{\varepsilon}}\left|\boldsymbol{u}-\boldsymbol{u}_{B}\right|^{2} d \boldsymbol{x} \leq C \int_{\Omega_{\varepsilon}}|\nabla \boldsymbol{u}|^{2} d \boldsymbol{x}
$$

where $B \subset \Omega \backslash\left\{\boldsymbol{x}_{1}, \ldots, \boldsymbol{x}_{N}\right), \boldsymbol{u}_{B}$ is defined as in (6.20) and the constant $C$ is independent of $\varepsilon$ (but may depend on $\Omega$ and $B$ ).

Proof. We follow closely the proof of Proposition 6.4. Fix $R>0$ such that $B_{R}\left(\boldsymbol{x}_{i}\right) \subset \Omega$ for any $i=1, \ldots, N$, and decompose $\Omega_{\varepsilon}$ as the union of the annuli $C_{\varepsilon, R}\left(\boldsymbol{x}_{i}\right)=B_{R}\left(\boldsymbol{x}_{i}\right) \backslash B_{\varepsilon}\left(\boldsymbol{x}_{i}\right)$ and its complement $\Omega^{\prime}$, which 


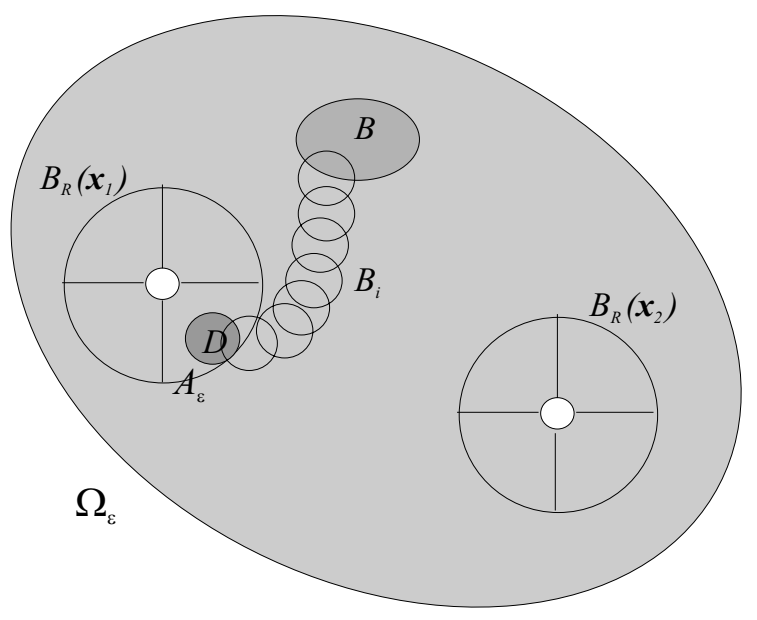

Figure 2: proof of Proposition 6.5: the figure shows the decomposition of the annuli $C_{\varepsilon, R}\left(\boldsymbol{x}_{i}\right)$ into sectors star-shaped with respect to a ball; the sector $A$ is star-shaped with respect to $D$. Also shown is a sequence of balls $B_{i}$ connecting $D$ to $B$.

is independent of $\varepsilon$. Since $\Omega^{\prime}$ and each annulus $C_{\varepsilon, R}\left(\boldsymbol{x}_{i}\right)$ have the cone property, each of them may be decomposed as the union of star-shaped domains with respect to some ball.

In particular, each annulus may be decomposed as the union of four $\pi / 2$-sectors, which are star-shaped with respect to some ball contained in the sector (cf. Figure 2).

Fix $\bar{\varepsilon}<R$, and let $A_{\bar{\varepsilon}}$ be one of these sectors, star-shaped with respect to the ball $D \subset A_{\bar{\varepsilon}}$, and notice that, for any $\varepsilon<\bar{\varepsilon}, A_{\varepsilon}$ is still star-shaped with respect to $D$ (cf. Figure 3 ). Applying now to $A_{\varepsilon}$ the construction in the proof of Proposition 6.4, we obtain the inequality (6.23), with $C_{1}$ and $C_{2}$ given by (6.25), and with $A$ replaced by $A_{\varepsilon}$. Now, notice that, since $A_{\varepsilon}$ is still star-shaped with respect to $D$, for any $\varepsilon<\bar{\varepsilon}$ we may choose the same sequence of balls $B_{i}$ connecting $D$ to $B$. Moreover, $\left|A_{\varepsilon}\right|=\frac{\pi}{4}\left(R^{2}-\varepsilon^{2}\right) \leq \pi R^{2} / 4=\left|A_{0}\right|$, and this implies that both $C_{1}$ and $C_{2}$ are bounded from above by a constant independent of $\varepsilon$. Proceeding now as in the proof of Proposition 6.4 we obtain the thesis.

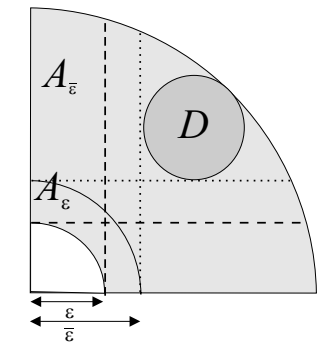

Figure 3: The sector $A_{\bar{\varepsilon}}$ is star-shaped with respect to the ball $D$ : if $\varepsilon<\bar{\varepsilon}$, then $A_{\varepsilon}$ remains star-shaped with respect to the same ball. 
We now turn to Korn's inequality: first of all we notice that, if $\Omega$ has the cone property then for all $\varepsilon>0 \Omega_{\varepsilon}$ has the cone property, the following version of this inequality holds (Friedrichs (1947), [29]):

Proposition 6.6. [Korn's inequality I] Let $\Omega \subset \mathbb{R}^{2}$ be an open bounded domain with the cone property, and $\boldsymbol{u} \in H^{1}\left(\Omega_{\varepsilon} ; \mathbb{R}^{2}\right)$ such that

$$
\int_{\Omega_{\varepsilon}}\left(\nabla \boldsymbol{u}-(\nabla \boldsymbol{u})^{\top}\right) d a=0
$$

then there exists a constant $K_{\varepsilon}$ such that

$$
\int_{\Omega_{\varepsilon}}|\nabla \boldsymbol{u}|^{2} d a \leq K_{\varepsilon} \int_{\Omega_{\varepsilon}}|\boldsymbol{E}(\boldsymbol{u})|^{2} d a .
$$

We continue to denote by $K_{\varepsilon}$ the infimum of all constants satisfying (6.29), and refer to it as Korn's constant for $\Omega_{\varepsilon}$. The following result shows that $K_{\varepsilon}$ is bounded from above independently of $\varepsilon$ as $\varepsilon \rightarrow 0$.

Proposition 6.7. Let $K_{\varepsilon}$ be Korn's constant for $\Omega_{\varepsilon}$ as defined in (6.29): then there exists a constant $K<\infty$, independent of $\varepsilon$ (but possibly depending on $\Omega$ ), such that

$$
K_{\varepsilon} \leq K
$$

for all $\varepsilon>0$.

Proof. Consider first the case $N=1$, and let $\boldsymbol{x}_{1}=\mathbf{0}$, so that $\Omega_{\varepsilon}=\Omega \backslash B_{\varepsilon}(\mathbf{0})$. The proof follows from two results of [11]. The first result states that the minimum value for Korn's constant of the annulus $C_{\varepsilon, R}(\mathbf{0})$ with internal radius $\varepsilon$ and external radius $R$ (under the constraint (6.28)) is

$$
4\left[1-\left(\frac{3 R^{2} \varepsilon^{2}}{R^{4}+R^{2} \varepsilon^{2}+\varepsilon^{4}}\right)\right]^{-1},
$$

which tends to Korn's constant for the circle $K=4$ as $\varepsilon \rightarrow 0$. The second result states that, if Korn's inequality (6.28)-(6.29) holds for two open bounded domains $\Omega_{1}$ and $\Omega_{2}$ such that $\left|\Omega_{1} \cap \Omega_{2}\right|>0$, then it also holds for $\Omega_{1} \cup \Omega_{2}$, and

$$
K_{12} \leq K_{1}+K_{2}+\frac{\min \left\{\left|\Omega_{1}\right|,\left|\Omega_{2}\right|\right\}}{\left|\Omega_{1} \cap \Omega_{2}\right|}\left(\sqrt{K_{1}}+\sqrt{K_{2}}\right)^{2}
$$

with $K_{12}, K_{1}$ and $K_{2}$ the Korn's constants of $\Omega_{1} \cup \Omega_{2}, \Omega_{1}$ and $\Omega_{2}$ respectively.

To prove (6.30), choose $R$ such that $2 R<d(\mathbf{0}, \partial \Omega)$, let $\Omega_{1}=C_{\varepsilon, 2 R}(\mathbf{0})$ and $\Omega_{2}=\Omega_{\varepsilon} \backslash C_{\varepsilon, R}(\mathbf{0})$, and apply (6.32): since $K_{2}$ is independent of $\varepsilon$ and, by (6.31), $K_{1} \rightarrow 4$ as $\varepsilon \rightarrow 0$, Korn's constant $K_{\varepsilon}=K_{12}$ is bounded from above, and the thesis follows for $N=1$.

When $N>1$, to obtain the thesis it is sufficient to iterate the above procedure: define

$$
\left\{\begin{array}{l}
\tilde{\Omega}_{0}:=\Omega \backslash\left(\cup_{i=1}^{N} B_{R}\left(\boldsymbol{x}_{i}\right)\right) \\
\vdots \\
\tilde{\Omega}_{i}:=\tilde{\Omega}_{i-1} \cup\left(B_{2 R}\left(\boldsymbol{x}_{i}\right) \backslash B_{\varepsilon}\left(\boldsymbol{x}_{i}\right)\right) \quad i=1, \ldots, N-1, \\
\vdots \\
\tilde{\Omega}_{N}:=\Omega_{\varepsilon} .
\end{array}\right.
$$


Applying (6.32) to each $\tilde{\Omega}_{i}$ we obtain

$$
\begin{aligned}
\tilde{K}_{i} & \leq \tilde{K}_{i-1}+\tilde{K}_{\varepsilon}+\frac{\min \left\{\left|\tilde{\Omega}_{i-1}\right|,\left|C_{\varepsilon, 2 R}\left(\boldsymbol{x}_{i}\right)\right|\right\}}{\left|\tilde{\Omega}_{i-1} \cap C_{\varepsilon, 2 R}\left(\boldsymbol{x}_{i}\right)\right|}\left(\sqrt{\tilde{K}_{i-1}}+\sqrt{\tilde{K}_{\varepsilon}}\right)^{2} \\
& =\tilde{K}_{i-1}+\tilde{K}_{\varepsilon}+\frac{4 R^{2}-\varepsilon^{2}}{3 R^{2}}\left(\sqrt{\tilde{K}_{i-1}}+\sqrt{\tilde{K}_{\varepsilon}}\right)^{2}
\end{aligned}
$$

where $\tilde{K}_{i}$ and $\tilde{K}_{\varepsilon}$ are Korn's constants for $\tilde{\Omega}_{i}$ and $C_{\varepsilon, 2 R}\left(\boldsymbol{x}_{i}\right)$ respectively. Using the relation $(a+b)^{2} \leq$ $2\left(a^{2}+b^{2}\right)$, and taking $\varepsilon=0$, this relation implies

$$
\tilde{K}_{i} \leq \frac{11}{3}\left(\tilde{K}_{i-1}+\tilde{K}_{\varepsilon}\right)
$$

which yields in turn

$$
\tilde{K}_{N} \leq\left(\frac{11}{3}\right)^{N} \tilde{K}_{0}+\left(\sum_{i=1}^{N}\left(\frac{11}{3}\right)^{i}\right) \tilde{K}_{\varepsilon} .
$$

Since $\tilde{K}_{\varepsilon}$ is given by (6.31) and is bounded from above, and $\tilde{K}_{0}$ is independent of $\varepsilon$, this relation shows that $K_{\varepsilon}=\tilde{K}_{N}$ is also bounded from above as $\varepsilon \rightarrow 0$.

Korn's inequality extends trivially to displacement fields $\boldsymbol{u}$ which do not satisfy (6.28):

Corollary 6.8. [Korn's inequality $\boldsymbol{I}^{\prime}$ ] Let $\Omega \subset \mathbb{R}^{2}$ be an open bounded domain with the cone property, and $\boldsymbol{u} \in H^{1}\left(\Omega_{\varepsilon} ; \mathbb{R}^{2}\right)$ : then there exists a constant $K$, independent of $\varepsilon$, such that

$$
\int_{\Omega_{\varepsilon}}|\nabla \boldsymbol{u}-\boldsymbol{W}|^{2} d a \leq K \int_{\Omega_{\varepsilon}}|\boldsymbol{E}(\boldsymbol{u})|^{2} d a,
$$

where

$$
\boldsymbol{W}=\frac{1}{2} \int_{\Omega_{\varepsilon}}\left(\nabla \boldsymbol{u}-(\nabla \boldsymbol{u})^{\top}\right) d a
$$

Combining (6.19), (6.29) and (6.30), we obtain finally the basic inequality

Proposition 6.9. [Korn's inequality II] Let $\boldsymbol{u} \in H^{1}\left(\Omega_{\varepsilon} ; \mathbb{R}^{2}\right)$ such that

$$
\int_{\Omega_{\varepsilon}}\left(\nabla \boldsymbol{u}-(\nabla \boldsymbol{u})^{\top}\right) d a=0
$$

then there exists a constant $K$, independent of $\varepsilon$, such that

$$
\int_{\Omega_{\varepsilon}}\left|\boldsymbol{u}-\boldsymbol{u}_{B}\right|^{2} d a+\int_{\Omega_{\varepsilon}}|\nabla \boldsymbol{u}|^{2} d a \leq K \int_{\Omega_{\varepsilon}}|\boldsymbol{E}(\boldsymbol{u})|^{2} d a
$$

where

$$
\boldsymbol{u}_{B}:=\frac{1}{|B|} \int_{B} \boldsymbol{u} d a,
$$

and $B$ is any fixed ball whose closure is contained in $\Omega_{\varepsilon}$, for all $\varepsilon$. 
We now show that the trace constant for $\Omega_{\varepsilon}$ may be chosen to be independent of $\varepsilon$.

Proposition 6.10. Let $\boldsymbol{u} \in H^{1}\left(\Omega_{\varepsilon} ; \mathbb{R}^{2}\right)$ : then there exists a positive constant $C$, independent of $\varepsilon$, such that

$$
\int_{\partial \Omega_{\varepsilon}}|\boldsymbol{u}|^{2} d s \leq C\left(\int_{\Omega_{\varepsilon}}|\boldsymbol{u}|^{2} d a+\int_{\Omega_{\varepsilon}}|\nabla \boldsymbol{u}|^{2} d a\right) .
$$

Proof. Let $R$ be such that $B_{R}\left(\boldsymbol{x}_{i}\right) \subset \Omega$ for each $i=1, \ldots, N$ and, for fixed $i$, let $(\varrho, \vartheta)$ be polar coordinates centered at $\boldsymbol{x}_{i}$ : for $\varepsilon<\varrho<R$ and $\boldsymbol{u} \in H^{1}\left(\Omega_{\varepsilon}, \mathbb{R}^{2}\right)$,

$$
\boldsymbol{u}(\varepsilon, \vartheta)=\boldsymbol{u}(\varrho, \vartheta)-\int_{\varepsilon}^{\varrho} \frac{\partial \boldsymbol{u}}{\partial \varrho}(r, \vartheta) d r
$$

form which it follows, by Hölder's inequality,

$$
|\boldsymbol{u}(\varepsilon, \vartheta)|^{2} \leq 2|\boldsymbol{u}(\varrho, \vartheta)|^{2}+2(R-\varepsilon) \int_{\varepsilon}^{R}\left|\frac{\partial \boldsymbol{u}}{\partial \varrho}(r, \vartheta)\right|^{2} d r
$$

Integrating with respect to $\vartheta$ yields

$$
\int_{0}^{2 \pi}|\boldsymbol{u}(\varepsilon, \vartheta)|^{2} d \vartheta \leq 2 \int_{0}^{2 \pi}|\boldsymbol{u}(\varrho, \vartheta)|^{2} d \vartheta+2(\varrho-\varepsilon) \int_{0}^{2 \pi} \int_{\varepsilon}^{\varrho}\left|\frac{\partial \boldsymbol{u}}{\partial \varrho}(r, \vartheta)\right|^{2} d r d \vartheta
$$

Multiplying by $\varepsilon$ gives

$$
\int_{\partial B_{\varepsilon}\left(\boldsymbol{x}_{i}\right)}|\boldsymbol{u}|^{2} d s \leq \frac{2 \varepsilon}{\varrho} \int_{\partial B_{\varrho}\left(\boldsymbol{x}_{i}\right)}|\boldsymbol{u}|^{2} d s+4(\varrho-\varepsilon) \int_{B_{\varrho}\left(\boldsymbol{x}_{i}\right) \backslash B_{\varepsilon}\left(\boldsymbol{x}_{i}\right)}|\nabla \boldsymbol{u}|^{2} d a .
$$

By integrating with respect to $\varrho$ we obtain

$$
\int_{\partial B_{\varepsilon}\left(\boldsymbol{x}_{i}\right)}|\boldsymbol{u}|^{2} d s \leq \frac{2}{R-\varepsilon} \int_{C_{\varepsilon, R}\left(\boldsymbol{x}_{i}\right)}|\boldsymbol{u}|^{2} d a+4(R-\varepsilon) \int_{C_{\varepsilon, R}\left(\boldsymbol{x}_{i}\right)}|\nabla \boldsymbol{u}|^{2} d a .
$$

Hence, for $\varepsilon$ sufficiently small, there exists a constant $C_{1}$ such that

$$
\int_{\partial B_{\varepsilon}\left(\boldsymbol{x}_{i}\right)}|\boldsymbol{u}|^{2} d s \leq C_{1}\left(\int_{\Omega_{\varepsilon}}|\boldsymbol{u}|^{2} d a+\int_{\Omega_{\varepsilon}}|\nabla \boldsymbol{u}|^{2} d a\right)
$$

for each $i=1, \ldots, N$. Now, let $C_{2}$ be the trace constant for $\Omega_{R}$, so that

$$
\int_{\partial \Omega}|\boldsymbol{u}|^{2} d s \leq C_{2}\left(\int_{\Omega_{R}}|\boldsymbol{u}|^{2} d a+\int_{\Omega_{R}}|\nabla \boldsymbol{u}|^{2} d a\right) \leq C_{2}\left(\int_{\Omega_{\varepsilon}}|\boldsymbol{u}|^{2} d a+\int_{\Omega_{\varepsilon}}|\nabla \boldsymbol{u}|^{2} d a\right) .
$$

Adding the expressions above we finally obtain the thesis.

Finally, we show that functions in $H^{1}\left(\Omega_{\varepsilon} ; \mathbb{R}^{2}\right)$ can be extended to $\Omega$ with extension constant independent of $\varepsilon$. 
Proposition 6.11. Let $\boldsymbol{u} \in H^{1}\left(\Omega_{\varepsilon} ; \mathbb{R}^{2}\right)$ : then $\boldsymbol{u}$ admits an extension $\hat{\boldsymbol{u}} \in H^{1}\left(\Omega, \mathbb{R}^{2}\right)$ such that

$$
\|\hat{\boldsymbol{u}}\|_{H^{1}\left(\Omega, \mathbb{R}^{2}\right)} \leq C\|\boldsymbol{u}\|_{H^{1}\left(\Omega_{\varepsilon} ; \mathbb{R}^{2}\right)}
$$

where the constant $C$ is independent of $\varepsilon$.

Proof. First notice that, using a partition of unity, we may assume that $\boldsymbol{u} \in H^{1}\left(\mathbb{R}^{2} \backslash B_{\varepsilon}(\mathbf{0}) ; \mathbb{R}^{2}\right)$, and $\boldsymbol{u}=0$ outside a compact in $\mathbb{R}^{2}$. Let

$$
\hat{\boldsymbol{u}}(\boldsymbol{x})= \begin{cases}\boldsymbol{u}\left(\frac{\varepsilon^{2}}{|\boldsymbol{x}|^{2}} \boldsymbol{x}\right) & \boldsymbol{x} \in B_{\varepsilon}(\mathbf{0}), \\ \boldsymbol{u}(\boldsymbol{x}) & \boldsymbol{x} \in \mathbb{R}^{2} \backslash B_{\varepsilon}(\mathbf{0}),\end{cases}
$$

so that $\hat{\boldsymbol{u}}(\boldsymbol{x})=\boldsymbol{u}(\boldsymbol{x})$ when $|\boldsymbol{x}|=\varepsilon$. Then

$$
\begin{aligned}
\int_{B_{\varepsilon}(\mathbf{0})}|\hat{\boldsymbol{u}}(\boldsymbol{x})|^{2} d a & =\int_{B_{\varepsilon}(\mathbf{0})}\left|\boldsymbol{u}\left(\frac{\varepsilon^{2}}{|\boldsymbol{x}|^{2}} \boldsymbol{x}\right)\right|^{2} d a=\int_{\mathbb{R}^{2} \backslash B_{\varepsilon}(\mathbf{0})} \frac{\varepsilon^{4}}{|\boldsymbol{x}|^{4}}|\boldsymbol{u}(\boldsymbol{x})|^{2} d a \\
& \leq \int_{\mathbb{R}^{2} \backslash B_{\varepsilon}(\mathbf{0})}|\boldsymbol{u}(\boldsymbol{x})|^{2} d a
\end{aligned}
$$

since the modulus of the Jacobian of the transformation $\boldsymbol{x} \rightarrow \varepsilon^{2} \boldsymbol{x} /|\boldsymbol{x}|^{2}$ is $|J|=\varepsilon^{4} /|\boldsymbol{x}|^{4}$, and $\varepsilon^{4} /|\boldsymbol{x}|^{4} \leq 1$ for $|\boldsymbol{x}| \geq \epsilon$. Also, notice that

$$
\nabla \hat{\boldsymbol{u}}(\boldsymbol{x})=\nabla \boldsymbol{u}\left(\frac{\varepsilon^{2}}{|\boldsymbol{x}|^{2}} \boldsymbol{x}\right)\left[\frac{\varepsilon^{2}}{|\boldsymbol{x}|^{2}}\left(\mathbf{1}-\frac{2}{|\boldsymbol{x}|^{2}} \boldsymbol{x} \otimes \boldsymbol{x}\right)\right]
$$

and

$$
\left|\mathbf{1}-\frac{2}{|\boldsymbol{x}|^{2}} \boldsymbol{x} \otimes \boldsymbol{x}\right|^{2}=1
$$

Hence,

$$
\begin{aligned}
\int_{B_{\varepsilon}(\mathbf{0})}|\nabla \hat{\boldsymbol{u}}(\boldsymbol{x})|^{2} d a & \leq M \int_{B_{\varepsilon}(\mathbf{0})} \frac{\varepsilon^{4}}{|\boldsymbol{x}|^{4}}\left|\nabla \boldsymbol{u}\left(\frac{\varepsilon^{2}}{|\boldsymbol{x}|^{2}} \boldsymbol{x}\right)\right|^{2} d a \\
& =M \int_{\mathbb{R}^{2} \backslash B_{\varepsilon}(\mathbf{0})} \frac{\varepsilon^{4}}{|\boldsymbol{x}|^{4}} \frac{|\boldsymbol{x}|^{4}}{\varepsilon^{4}}|\nabla \boldsymbol{u}(\boldsymbol{x})|^{2} d a \\
& \leq M \int_{\mathbb{R}^{2} \backslash B_{\varepsilon}(\mathbf{0})}|\nabla \boldsymbol{u}(\boldsymbol{x})|^{2} d a,
\end{aligned}
$$

with $M$ a positive constant independent of $\varepsilon$. Since $\hat{\boldsymbol{u}}=\boldsymbol{u}$ on $\mathbb{R}^{2} \backslash B_{\varepsilon}(\mathbf{0})$, it follows that there exists a constant $C$ independent of $\varepsilon$ such that

$$
\|\hat{\boldsymbol{u}}\|_{H^{1}\left(\mathbb{R}^{2}, \mathbb{R}^{2}\right)} \leq C\|\boldsymbol{u}\|_{H^{1}\left(\mathbb{R}^{2} \backslash B_{\varepsilon}(\mathbf{0}) ; \mathbb{R}^{2}\right)}
$$

which implies the thesis. 


\section{Acnowledgements}

The research of G. Leoni was partially supported by the National Science Foundation under Grant No. DMS-0405423. The authors wish to thank A. Garroni for interesting conversations on the subject of this paper, the Center for Nonlinear Analysis (NSF Grant No. DMS-9803791), Carnegie Mellon University, Pittsburgh, PA, USA, and the Italian MIUR (Cofin 2002 "Modelli matematici in Scienza dei Materiali") for their support during the preparation of this paper.

\section{References}

[1] E.C. Aifantis, The physics of plastic deformation, International Journal of Plasticity 3 (1987), 211-247.

[2] G. Alberti, S. Baldo and G. Orlandi, Variational convergence for functionals of Ginzburg-Landau type to appear in Indiana Univ. Math. J.

[3] R.J. Asaro, Micromechanics of crystals and polycrystals, Advances in Applied Mechanics 23 (1983), $1-115$.

[4] R.J.Asaro and A. Needleman, Texture development and strain hardening in rate dependent polycrystals, Acta metallurgica 33 (1985), 923-953.

[5] F. Bethuel, H. Brezis, B.D. Coleman and F. Hélein, Bifurcation analysis of minimizing harmonic maps describing the equilibrium of nematic phases between cylinders, Arch. Rational Mech. Anal. 118 (1992), 149-168.

[6] F. Bethuel, H. Brezis and F. Hélein, Ginzburg-Landau vortices, Birkhuser, Basel (1994).

[7] F. Bethuel, H. Brezis and G. Orlandi, Asymptotics for the Ginzburg-Landau equation in arbitrary dimensions, J. Funct. Anal. 186 (2001), 432-520.

[8] P. Cermelli and M.E. Gurtin, The motion of screw dislocations in materials undergoing anti-plane shear: glide, cross-slip, fine cross-slip, Arch. Rat. Mech. Anal. 148 (1999), 3-52.

[9] P. Cermelli, M.E. Gurtin and G. Leoni, Energies for incoherent films: an analytical approach, Interfaces and Free Boundaries 1 (1999), 81-105.

[10] S. Conti, I. Fonseca, and G. Leoni, $\Gamma$-convergence result for the two-gradient theory of phase transitions, Commun. Pure Appl. Math. 55 (2002), 857-936.

[11] C.M. Dafermos, Some remarks on Korn's inequality, Zeitschrift für Angewandte Mathematik und Physik 19 (1968), 913-920.

[12] R. Dautray and J.L. Lions, Mathematical analysis and numerical methods for science and technology, Vol. 3, Springer, Berlin (1988).

[13] J.L. Ericksen, Remarks concerning forces on line defect, Zeitschrift für Angewandte Mathematik und Physik 46 (1995), S247-S271.

[14] J.D. Eshelby, The force on elastic singularity, Phil. Trans. R. Soc. London A244 (1951), 87-112. 
[15] J.D. Eshelby, The continuum theory of lattice defects, in Solid State Physics (eds. F. Seitz and D. Turnbull) 3 (1956), 79-144.

[16] L. C. Evans, Weak convergence methods for nonlinear partial differential equations, Regional Conference Series in Mathematics, 74. American Mathematical Society, Providence (1990).

[17] N. A. Fleck and J. W. Hutchinson, A phenomenological theory for strain gradient effects in plasticity, Journal of the Mechanics and Physics of Solids 41 (1993), 1825-1857.

[18] N. A. Fleck and J. W. Hutchinson, Strain gradient plasticity, Advances in Applied Mechanics 33 (1997), 295-361.

[19] N. A. Fleck, G.M. Muller, M.F. Ashby and J.W. Hutchinson, Strain gradient plasticity: theory and experiment, Acta metallurgica 42 (1994), 475-487.

[20] D. François, A. Pineau and A. 0 Zaoui, Mechanical behaviour of materials I, Kluwer, Dordrecht (1998).

[21] K. O. Friedrichs, On the boundary-value problems of the theory of elasticity and Korn's inequality, Annals of Mathematics 48/2 (1947), 441-471.

[22] D. Gilbarg and N. Trudinger, Elliptic partial differential equations of second order, 2nd edition, Springer-Verlag, Berlin, (1983).

[23] M. E. Gurtin, Configurational forces as basic concepts of continuum physics, Springer-Verlag, New York (2000).

[24] M. E. Gurtin, On the plasticity of single crystals: free energy, microforces, plastic-strain gradients, Journal of the Mechanics and Physics of Solids 48 (2000), 989-1036.

[25] M. E. Gurtin, A gradient theory of single-crystal viscoplasticity that accounts for geometrically necessary dislocations, Journal of the Mechanics and Physics of Solids 50 (2002), 5-32.

[26] R.L. Jerrard and H.M. Soner, Dynamics of Ginzburg-Landau vortices, Arch. Ration. Mech. Anal. 142 (1998), 99-125.

[27] R.L. Jerrard and H.M. Soner, The Jacobian and the Ginzburg-Landau energy, Calc. Var. Partial Differential Equations 14 (2002), 151-191.

[28] J.P. Hirth and J. Lothe, Theory of dislocations, 2nd Ed., Wiley (1982).

[29] C. O. Horgan, Korn's inequalities and their applications in continuum mechanics, SIAM Review $\mathbf{3 7} / 4$ (1995), 491-511.

[30] J. Kratochvíl and M. Saxlová, On the physical foundations of plasticity, Meccanica 31 (1996), 495-506.

[31] D. Kuhlmann-Wilsdorf, Theory of plastic deformation: properties of low energy dislocation structures, Materials Science and Engineering A113 (1989), 1-41.

[32] F.-H. Lin and C. Liu, Static and dynamic theories of liquid crystals, J. Partial Differential Equations 14 (2001), 289-330. 
[33] F.-H. Lin and J. X. Xin, On the dynamical law of the Ginzburg-Landau vortices on the plane, Comm. Pure Appl. Math. 52 (1999), 1189-1212.

[34] N.I. Muskhelishvili, Some basic problems in the mathematical theory of elasticity, Noordhoff, Groningen (1963).

[35] F.R.N. Nabarro, Theory of crystal dislocations, Clarendon Press, Oxford (1967).

[36] P. M. Naghdi and A.R. Srinivasa, A dynamical theory of structured solids, Philosophical Transactions of the Royal Society of London 345A (1993), 425-458.

[37] M. Peach and J.S. Köhler, The forces exerted on dislocations and the stress field produced by them, Phys. Rev. 80 (1950), 436-439.

[38] T. Suzuki, S. Takeuchi and H. Yoshinaga, Dislocation dynamics and plasticity, Springer-Verlag, Berlin (1985).

[39] C. Teodosiu, A dynamic theory of dislocations and its application to the theory of the elasto-plastic continuum, in: J. A. Simmons, R. de Wit and R. Bollough (Eds.), Proceedings of the Conference on Fundamental Aspects of Dislocation Theory, 1969. Natl. Bur. Stand. Spec. Publ. 317/2 (1970), 837-876.

Dipartimento di Matematica, Università di Torino,

Via Carlo Alberto 10,

I-10123 Torino, Italy

paolo. cermelli@unito.it

Department of Mathematical Sciences,

Carnegie Mellon University,

Pittsburgh, PA 15213, USA

giovanni@andrew. cmu . edu 